\title{
Asymptotics for the heat content of a planar region with a fractal polygonal boundary
}

\author{
M. van den Berg \\ School of Mathematics \\ University of Bristol, University Walk \\ Bristol BS8 1TW, United Kingdom \\ F. den Hollander \\ Mathematical Institute \\ University of Nijmegen, Toernooiveld 1 \\ 6525 ED Nijmegen, The Netherlands
}

February 18, 1997

\begin{abstract}
Let $k \geq 3$ be an integer. For $0<s<1$, let $D_{s} \subset \mathbb{R}^{2}$ be the set that is constructed iteratively as follows. Take a regular open $k$-gon with sides of unit length, attach regular open $k$-gons with sides of length $s$ to the middles of the edges, and so on. At each stage of the iteration the $k$-gons that are added are a factor $s$ smaller than the previous generation and are attached to the outer edges of the family grown so far. The set $D_{s}$ is defined to be the interior of the closure of the union of all the $k$-gons. It is easy to see that there must exist some $s_{k}>0$ such that no $k$-gons overlap if and only if $0<s \leq s_{k}$. We derive an explicit formula for $s_{k}$.

The set $D_{s}$ is open, bounded, connected and has a fractal polygonal boundary. Let $E_{D_{s}}(t)$ denote the heat content of $D_{s}$ at time $t$ when $D_{s}$ initially has temperature 0 and $\partial D_{s}$ is kept at temperature 1 . We derive the complete short-time expansion of $E_{D_{s}}(t)$ up to terms that are exponentially small in $1 / t$. It turns out that there are three regimes, corresponding to $0<s<1 /(k-1), s=1 /(k-1)$ respectively $1 /(k-1)<s \leq s_{k}$. For $s \neq$ $1 /(k-1)$ the expansion has the form $E_{D_{s}}(t)=p_{s}(\log t) t^{1-\frac{d_{s}}{2}}+A_{s} t^{\frac{1}{2}}+B t+O\left(e^{-\frac{r_{s}}{t}}\right)$, where $p_{s}$ is a $\log \left(1 / s^{2}\right)$-periodic function, $d_{s}=\log (k-1) / \log (1 / s)$ is a similarity dimension, $A_{s}$ and $B$ are constants related to the edges respectively vertices of $D_{s}$, and $r_{s}$ is an error exponent. For $s=1 /(k-1)$ the $t^{\frac{1}{2}}$-term carries an additional $\log t$.
\end{abstract}

AMS 1991 subject classifications. 35K05, 28A80, 60J65.

Key words and phrases. Fractal polygon, heat equation, Brownian motion. Running title: Heat content of a fractal polygon.

* Research supported by the British Council and the Netherlands Organization for Scientific Research, joint scientific research project JRP208/171. 


\section{Contents}




\section{Introduction and main results: Theorems 1-3}

For $m \geq 2$ integer, let $D$ be an open set in Euclidean space $\mathbb{R}^{m}$ with boundary $\partial D$. Let $u_{D}:(D \cup \partial D) \times[0, \infty) \rightarrow \mathbb{R}$ be the unique (weak) solution of the heat equation

$$
\begin{array}{ll}
\frac{\partial}{\partial t} u_{D}=\Delta u_{D} & (x \in D, t>0) \\
u_{D}(x ; 0)=0 & (x \in D) \\
u_{D}(x ; t)=1 & (x \in \partial D, t>0),
\end{array}
$$

i.e., $D$ initially has temperature 0 and heats up because $\partial D$ is kept at temperature 1 . The total heat content of $D$ at time $t$ is

$$
E_{D}(t)=\int_{D} d x u_{D}(x ; t)
$$

The asymptotic behaviour of $E_{D}(t)$ as $t \downarrow 0$ is well understood if $D$ is bounded and $\partial D$ is smooth (see [3,4]). Moreover, for $m=2$ and for $D$ bounded and connected and $\partial D$ a finite polygonal, it is shown in [1] that

$$
E_{D}(t)=\frac{2}{\pi^{\frac{1}{2}}}|\partial D| t^{\frac{1}{2}}-t \sum_{i=1}^{N} c\left(\gamma_{i}\right)+O\left(e^{-\frac{r}{t}}\right) \quad(t \downarrow 0) .
$$

Here, $|\partial D|$ is the length of $\partial D, \gamma_{1}, \ldots, \gamma_{N}$ are the interior angles at the vertices of $\partial D$,

$$
c(\gamma)=4 \int_{0}^{\infty} d \zeta \frac{\sinh ((\pi-\gamma) \zeta)}{\sinh (\pi \zeta) \cosh (\gamma \zeta)}
$$

is an angle function, and $r$ is a strictly positive constant depending on $D$. Note that the leading term in (1.3) is proportional to the length of the boundary, while the correction term gives a negative contribution for non-reflex angles $\left(0<\gamma_{i}<\pi\right)$ and a positive contribution for reflex angles $\left(\pi<\gamma_{i}<2 \pi\right)$. Contrary to the case where $\partial D$ is smooth, there are no polynomial terms beyond order $t$.

In this paper we consider the problem of finding the asymptotic behaviour of $E_{D_{s}}(t)$ as $t \downarrow 0$ for a specific region $D_{s} \subset \mathbb{R}^{2}$ with a fractal polygonal boundary. This region is constructed as follows (see Fig. 1). Let $k \geq 3$ be an integer. Let $Q_{0}$ be a regular open $k$-gon with $\left|\partial Q_{0}\right|=k$ (sides of unit length). Attach $k$ regular open $k$-gons $Q_{1,1}, \ldots, Q_{1, k}$ with $\left|\partial Q_{1, i}\right|=k s$ to the middles of the $k$ edges of $Q_{0}(0<s<1$ is the scaling factor $)$. Proceed by induction and for $j=2,3, \ldots$ attach $N(j)=k(k-1)^{j-1}$ regular open $k$-gons $Q_{j, 1}, \ldots, Q_{j, N(j)}$ with $\left|\partial Q_{j, i}\right|=k s^{j}$ to the middles of the $k-1$ outer edges of $Q_{j-1,1}, \ldots, Q_{j-1, N(j-1)}$. In Section 2 we shall see that there exists an $s_{k}>0$ such that no $k$-gons overlap if and only if $0<s \leq s_{k}$, and we shall derive an explicit expression for $s_{k}$. The region $D_{s}$ is now defined as

$$
D_{s}=\text { interior }\left\{\overline{Q_{0} \cup\left[\cup_{j \geq 1} \cup_{1 \leq i \leq N(j)} Q_{j, i}\right]}\right\} \quad\left(k \geq 3,0<s \leq s_{k}\right) .
$$

The set $D_{s}$ is open, bounded, simply connected and has volume ${ }^{1}$

$$
\left|D_{s}\right|=\frac{1+s^{2}}{1-(k-1) s^{2}}\left|Q_{0}\right|
$$

\footnotetext{
${ }^{1}$ At the end of Section 2 we shall see that $s_{k}^{2}<1 /(k-1)$, so $\left|D_{s}\right|<\infty$ for all $0<s \leq s_{k}$. This is no surprise, because $D_{s_{k}}$ is bounded and all its $k$-gons are disjoint.
} 
where $\left|Q_{0}\right|$ is the volume of the basic $k$-gon:

$$
\left|Q_{0}\right|=\frac{1}{4} k \cot \left(\frac{\pi}{k}\right)
$$

The boundary $\partial D_{s}$ is not strictly fractal self-similar. Still, it has an interior Minkowski dimension (see [5]) given by

$$
d\left(\partial D_{s}\right)=\max \left\{1, d_{s}\right\}
$$

where $0<d_{s}<2$ is a similarity dimension given by

$$
d_{s}=\frac{\log (k-1)}{\log \left(\frac{1}{s}\right)}
$$

The length of $\partial D_{s}$ is

$$
\left|\partial D_{s}\right|= \begin{cases}\frac{k(1-s)}{1-(k-1) s} & \left(0<s<\frac{1}{k-1}\right) \\ \infty & \left(\frac{1}{k-1} \leq s \leq s_{k}\right) .\end{cases}
$$

Moreover, one can show that $\partial D_{s}$ has finite $d\left(\partial D_{s}\right)$-dimensional upper Minkowski content and strictly positive $d\left(\partial D_{s}\right)$-dimensional lower Minkowski content (see [5]). Therefore, $D_{s}$ being simply connected, [5] Corollary 1.5 implies that for $1 /(k-1)<s \leq s_{k}$ there exist constants $0<C<\infty$ and $0<t_{0}<\infty$ such that

$$
\frac{1}{C} t^{1-\frac{d_{s}}{2}} \leq E_{D_{s}}(t) \leq C t^{1-\frac{d_{s}}{2}} \quad\left(0 \leq t \leq t_{0}\right)
$$

As far as we know, the only non-trivial example for which a more detailed analysis is available is the triadic von Koch snowflake $K$. Namely, in [7] the existence is proved of two ( $\log 9)$-periodic functions $p$ and $q$ such that

$$
E_{K}(t)=p(\log t) t^{1-\frac{\log 2}{\log 3}}-q(\log t) t+O\left(e^{-\frac{1}{1152 t}}\right) .
$$

Expansion (1.12) resembles (1.3) for finite polygons in that both have an exponential remainder term as well as a term of order $t$.

Our main motivation for studying the heat content of the regions $D_{s}\left(k \geq 3,0<s \leq s_{k}\right)$ defined above is that by varying both $k$ and $s$ we shall be able to obtain some insight into the structure of the asymptotic expansion. Indeed, the expansion obtained below (for three different $s$-regimes) contains three terms, two of which are computed explicitly and the third of which contains a periodic function like the ones appearing in (1.12).

In the three theorems below,

$$
\gamma_{k}=\pi-\frac{2 \pi}{k}
$$

denotes the interior angle of the regular $k$-gons, $c$ is the angle function defined in (1.4), $d_{s}$ is the similarity dimension defined in (1.9), and $r_{s}$ is an error exponent given by

$$
r_{s}= \begin{cases}\frac{s^{4}}{64}(3-\sqrt{5})(2-\sqrt{3}) & (k=3) \\ \frac{s^{4}}{8}(3-2 \sqrt{2}) & (k=4) \\ \frac{s^{4}}{16} \sin ^{2}\left(\frac{\pi}{k}\right) & (k \geq 5) .\end{cases}
$$

All expansions are for $t \downarrow 0$. 
Theorem 1 Let $k=3, \ldots, 8$ and $1 /(k-1)<s \leq s_{k}$. Then

$$
E_{D_{s}}(t)=p_{s}(\log t) t^{1-\frac{d_{s}}{2}}+\frac{2}{\pi^{\frac{1}{2}}} \frac{k(1-s)}{1-(k-1) s} t^{\frac{1}{2}}+\frac{2 k}{k-2} c\left(\pi+\gamma_{k}\right) t+O\left(e^{-\frac{r_{s}}{t}}\right),
$$

with $p_{s}$ a $\log \left(1 / s^{2}\right)$-periodic, continuous and strictly positive function.

Theorem 2 Let $k=3, \ldots, 8$ and $s=1 /(k-1)$. Then

$E_{D_{\frac{1}{k-1}}}(t)=\frac{1}{\pi^{\frac{1}{2}}} \frac{k(k-2)}{(k-1) \log (k-1)} t^{\frac{1}{2}} \log \left(\frac{1}{t}\right)+p_{\frac{1}{k-1}}(\log t) t^{\frac{1}{2}}+\frac{2 k}{k-2} c\left(\pi+\gamma_{k}\right) t+O\left(e^{-\frac{r_{1} /(k-1)}{t}}\right)$,

with $p_{\frac{1}{k-1}} a \log \left((k-1)^{2}\right)$-periodic and continuous function.

Theorem 3 Let $k=3, \ldots, 8$ and $0<s<1 /(k-1)$ or $k=9,10, \ldots$ and $0<s \leq s_{k}$. Then

$$
E_{D_{s}}(t)=\frac{2}{\pi^{\frac{1}{2}}} \frac{k(1-s)}{1-(k-1) s} t^{\frac{1}{2}}+p_{s}(\log t) t^{1-\frac{d_{s}}{2}}+\frac{2 k}{k-2} c\left(\pi+\gamma_{k}\right) t+O\left(e^{-\frac{r_{s}}{t}}\right),
$$

with $p_{s}$ a $\log \left(1 / s^{2}\right)$-periodic and continuous function.

Note that (1.15) and (1.17) are identical except for the order of the first two terms. We shall see in Section 2 that $s_{k}<1 /(k-1)$ if and only if $k \geq 9$, which explains the various cases in Theorems 1-3.

\section{REMARKS:}

1. A small computation shows that $\max \left\{1, d_{s}\right\}$ is the interior Minkowski dimension of the edges of $\partial D_{s}$ (as stated in (1.8)) and that $d_{s}$ is the interior Minkowski dimension of the vertices of $\partial D_{s}$. For $1 /(k-1)<s \leq s_{k}$, the leading term in the expansion agrees with the earlier estimate (1.11). In this case $d_{s}>1$. For $0<s<1 /(k-1)$, on the other hand, the leading term in the expansion is the same as in (1.3) because $\left|\partial D_{s}\right|<\infty$ (recall $(1.10)$ ). In this case $d_{s}<1$. For $s=1 /(k-1)$, finally, the behavior is critical. In this case $d_{s}=1$.

2. Note that the coefficient of $t$ does not depend on $s$. This is because, unlike the edges, the angles at the vertices do not depend on $s$. It is remarkable that in this coefficient only $\pi+\gamma_{k}$ features and not $\gamma_{k}$. Apparently, only those angles in $\partial D_{s}$ where the $k$-gons make contact survive in the expansion.

3. The sign of the coefficient of $t$ is different from (1.3). Apparently, the term of order $t^{\frac{1}{2}}$ already contains part of the contribution of the angles at the vertices.

4. We conjecture that $p_{s}$ is non-constant. Heuristically this may be visualised by thinking of a 'heat front' that moves in from the boundary and that has a period $\log \left(1 / s^{2}\right)$ on a logarithmic time scale due to the discrete self-similar structure of $\partial D_{s}$ and the classical space-time scaling of heat conduction. Unfortunately, we have not been able to confirm this picture. As will become clear at the end of Section 4, the function $p_{s}$ is an infinite sum of scaled quantities and as such is a rather complicated object. 
The remainder of this paper is structured as follows. In Section 2 we compute the value of $s_{k}$. In Section 3 we derive some estimates on $u_{D_{s}}(x ; t)$ for points near a vertex of $\partial D_{s}$ and for points near an edge of $\partial D_{s}$ but not near a vertex. The contribution coming from points away from $\partial D_{s}$ is shown to be exponentially small in $1 / t$. In Section 4 we derive a functional equation for $E_{D_{s}}(t)$, from which we are able to draw out the expansions (1.15-1.17) and show that $p_{s}$ is $\log \left(1 / s^{2}\right)$-periodic. In Section 5 we prove that $p_{s}$ is continuous, which by periodicity implies that $p_{s}$ is bounded, and we derive upper and lower bounds on $p_{s}$. Finally, in Section 6 we give some results (without proofs) for the partition function of the Dirichlet problem associated with $D_{s}$. These are obtained along the lines of Sections 3-5.

\section{Computation of $s_{k}$ : Theorem 4}

It is easy to see that for all $k \geq 3$ there must exist some $s_{k}>0$ such that no $k$-gons in the iterative construction of $D_{s}$ overlap if and only if $0<s \leq s_{k}$. The following theorem gives an explicit (and surprisingly simple) formula for $s_{k}$.

Theorem 4 All $k$-gons are disjoint if and only if $0<s \leq s_{k}$ with

$$
s_{k}=\frac{1}{\eta_{k}}\left(\sqrt{1+\eta_{k}^{2}}-1\right)
$$

where

$$
\begin{aligned}
& k \text { even: } \eta_{k}=1-\cos \left(\frac{2 \pi}{k}\right) \\
& k \text { odd: } \quad \eta_{k}=2 \frac{1-\cos \left(\frac{2 \pi}{k}\right)}{1+\cos \left(\frac{\pi}{k}\right)}=4\left[1-\cos \left(\frac{\pi}{k}\right)\right] .
\end{aligned}
$$

The first few values of $s_{k}$ are:

$$
\begin{aligned}
& s_{3}=\frac{1}{2}(\sqrt{5}-1) \quad=0.6180 \ldots\left(\eta_{3}=2\right) \\
& s_{4}=\sqrt{2}-1 \quad=0.4142 \ldots \quad\left(\eta_{4}=1\right) \\
& s_{5}=\frac{1}{4}(\sqrt{30+6 \sqrt{5}}-3-\sqrt{5})=0.3382 \ldots \quad\left(\eta_{5}=3-\sqrt{5}\right) \\
& s_{6}=\sqrt{5}-2 \quad=0.2360 \ldots \quad\left(\eta_{6}=\frac{1}{2}\right) .
\end{aligned}
$$

Proof. Because of the self-similar growing procedure, all $k$-gons are disjoint if and only if:

(I) The $k$-gons grown from one edge of $Q_{0}$ do not intersect the line through this edge.

(II) The $k$-gons grown from two neighbouring edges of $Q_{0}$ do not intersect the line that bisects the angle between these edges.

In order to investigate (I) and (II) we first need to locate the vertices of the $k$-gons.

1. Location of the vertices. Pick an edge of $Q_{0}$ and place this edge vertically, with its middle at the origin and with $Q_{0}$ in the left half plane. We begin by writing down a formula for the centres of all the $k$-gons grown from this edge. For this is will be convenient to map $\mathbb{R}^{2}$ to the complex plane: $\left(x_{1}, x_{2}\right) \rightarrow x_{1}+i x_{2}$.

The first generation consists of one $k$-gon, say $Q_{1,1}$, with sides of length $s$ and centre at

$$
c_{1}=L s e^{i 0}
$$


where $L=\frac{1}{2} \cot \left(\frac{\pi}{k}\right)$ is the inner radius of the basic $k$-gon $Q_{0}$ (i.e., the distance from the centre to the middles of the edges). The second generation consists of $k-1$ scaled $k$-gons, say $Q_{2,1}, \ldots, Q_{2, k-1}$, whose centres are located at positions

$$
c_{2}^{j_{1}}=c_{1}+L\left(s+s^{2}\right) e^{i\left(\pi-j_{1} \frac{2 \pi}{k}\right)} \quad\left(j_{1}=1, \ldots, k-1\right) .
$$

Namely, the middles of the $k-1$ outer edges of $Q_{1,1}$ lie in the directions $\pi-j_{1} \frac{2 \pi}{k}$ ( $j_{1}=$ $1, \ldots, k-1)$ relative to the direction of the centre of $Q_{1,1}$. The backward direction with $j_{1}=0$ is excluded, because this is where $Q_{1,1}$ is attached to $Q_{0}$. Note that $L\left(s+s^{2}\right)$ is the sum of the inner radii of $Q_{1,1}$ and $Q_{2,1}, \ldots, Q_{2, k-1}$. Similarly, the centres of the $(k-1)^{2}$ scaled $k$-gons in the third generation are located at positions

$$
c_{3}^{j_{1}, j_{2}}=c_{2}^{j_{1}}+L\left(s^{2}+s^{3}\right) e^{i\left[\left(\pi-j_{1} \frac{2 \pi}{k}\right)+\left(\pi-j_{2} \frac{2 \pi}{k}\right)\right]} \quad\left(j_{1}, j_{2}=1, \ldots, k-1\right) .
$$

Inductively, we thus find that the centres of the $(N+1)$-st generation are

$$
c_{N+1}^{j_{1}, \ldots, j_{N}}=L\left[s+\sum_{n=1}^{N}\left(s^{n}+s^{n+1}\right) e^{i \pi \sum_{m=1}^{n} \alpha\left(j_{m}\right)}\right] \quad\left(j_{1}, \ldots, j_{N}=1, \ldots, k-1\right),
$$

where we abbreviate $\alpha(j)=1-j \frac{2}{k}$.

Having found (2.7), we can now write down a formula for the vertices of the $k$-gons. Let $L^{\prime}$ be the outer radius of the basic $k$-gon $Q_{0}$ (i.e., the distance from the centre to the vertices), which equals $L^{\prime}=L / \cos \left(\frac{\pi}{k}\right)$. Then the vertices of the $k$-gon with centre $c_{N+1}^{j_{1}, \ldots, j_{N}}$ are located at positions

$$
v_{N+1}^{j_{1}, \ldots, j_{N}}\left(j_{N}^{*}\right)=c_{N+1}^{j_{1}, \ldots, j_{N}}+L^{\prime} s^{N+1} e^{i \pi\left[\sum_{m=1}^{N} \alpha\left(j_{m}\right)+\alpha\left(j_{N}^{*}\right)+\frac{1}{k}\right]} \quad\left(j_{N}^{*}=0,1, \ldots, k-1\right) .
$$

The angles in the last term are $\frac{\pi}{k}-j_{N}^{*} \frac{2 \pi}{k}\left(j_{N}^{*}=0,1, \ldots, k-1\right)$ relative to the direction $\pi \sum_{m=1}^{N} \alpha\left(j_{m}\right)+\pi$ of the previous centre.

2. Verification of (I). For the $k$-gons not to intersect the vertical axis (which is the line through the edge from which they are grown) the following condition is necessary and sufficient:

$$
\operatorname{Re}\left(v_{N+1}^{j_{1}, \ldots, j_{N}}\left(j_{N}^{*}\right)\right) \geq 0 \text { for all indices. }
$$

Substituting (2.7-2.8) into (2.9) and dividing by $L$, we obtain for condition (I)

$$
\begin{aligned}
s & +(1+s) \sum_{n=1}^{N} s^{n} \cos \left(\pi \sum_{m=1}^{n} \alpha\left(j_{m}\right)\right) \\
& +s^{N+1} \frac{1}{\cos \left(\frac{\pi}{k}\right)} \cos \left(\pi\left[\sum_{m=1}^{N} \alpha\left(j_{m}\right)+\alpha\left(j_{N}^{*}\right)+\frac{1}{k}\right]\right) \geq 0 \text { for all indices. }
\end{aligned}
$$

Define

$$
x_{N}=\inf _{\left\{j_{1}, \ldots, j_{N} ; j_{N}^{*}\right\}} \text { l.h.s. }(2.10) \quad(N \geq 0) .
$$

Then $L x_{N}$ is the distance to the vertical axis of the left-most vertex in the $(N+1)$-st generation. We must show that $x_{N} \geq 0$ for all $N \geq 0$ if and only if $0<s \leq s_{k}$ with $s_{k}$ given by $(2.1-2.2)$. 
Trivially, $x_{0}=0$ because the 1 -st $k$-gon is attached to the vertical axis (the infimum is attained at $\left.j_{0}^{*}=0\right)$. For $N \geq 1$, on the other hand, the infimum is attained when

$$
\begin{aligned}
& \sum_{m=1}^{n} \alpha\left(j_{m}\right)(1 \leq n \leq N) \text { and } \sum_{m=1}^{N} \alpha\left(j_{m}\right)+\alpha\left(j_{N}^{*}\right)+\frac{1}{k} \\
& \text { are as close as possible to } 1(\bmod 2) .
\end{aligned}
$$

This occurs (not necessarily uniquely) when

$$
\begin{array}{ll}
N=1: & j_{1}=1 \\
& j_{1}^{*}=\left\lfloor\frac{k}{2}\right\rfloor \\
N \geq 2: & j_{1}=1, j_{2}=\left\lceil\frac{k}{2}\right\rceil-1, \\
& j_{m}=\left\lfloor\frac{k}{2}\right\rfloor(3 \leq m \leq N \text { odd }), j_{m}=\left\lceil\frac{k}{2}\right\rceil(3 \leq m \leq N \text { even }), \\
& j_{N}^{*}=\left\lceil\frac{k}{2}\right\rceil .
\end{array}
$$

Hence we find

$$
\begin{array}{ll}
k \text { even: } & x_{N}=s-(1+s)\left[s \cos \left(\frac{2 \pi}{k}\right)+\sum_{n=2}^{N} s^{n}\right]-s^{N+1} \\
& (N \geq 1) \\
k \text { odd: } \quad & x_{N}=s-(1+s)\left[s \cos \left(\frac{2 \pi}{k}\right)+\sum_{n=2, \text { even }}^{N} s^{n} \cos \left(\frac{\pi}{k}\right)+\sum_{n=2, \text { odd }}^{N} s^{n}\right]-s^{N+1} \\
& (N \geq 1 \text { even }) \\
& x_{N}=s-(1+s)\left[s \cos \left(\frac{2 \pi}{k}\right)+\sum_{n=2, \text { even }}^{N} s^{n} \cos \left(\frac{\pi}{k}\right)+\sum_{n=2, \text { odd }}^{N} s^{n}\right]-s^{N+1} \frac{1}{\cos \left(\frac{\pi}{k}\right)} \\
& (N \geq 1 \text { odd }) .
\end{array}
$$

Our next step is to look at differences. From (2.14) we easily deduce the following:

$$
\begin{array}{lll}
k \text { even: } & x_{N}-x_{N+1}=2 s^{N+2} & (N \geq 1) \\
k \text { odd: } & x_{N}-x_{N+2}=s^{N+2}(1+s)\left(1+\cos \left(\frac{\pi}{k}\right)\right) & \\
& x_{N}-x_{N+2}=s^{N+1}(1+s) \frac{1+\cos \left(\frac{\pi}{k}\right)}{\cos \left(\frac{\pi}{k}\right)}\left\{s-\left(1-\cos \left(\frac{\pi}{k}\right)\right)\right\} & (N \geq 1 \text { even })
\end{array}
$$

From this we see that $\left(x_{N}\right)_{N \geq 1}$ is always decreasing when $k$ is even, always decreasing along the even integers when $k$ is od $\bar{d}$, and decreasing or increasing along the odd integers (depending on $s$ ) when $k$ is odd. Consequently, we have $x_{N} \geq 0$ for all $N \geq 1$ if and only if

$$
\begin{array}{ll}
k \text { even: } & x_{\infty} \geq 0 \\
k \text { odd: } & x_{\infty} \geq 0, x_{1} \geq 0 .
\end{array}
$$

Hence it remains to investigate (2.16).

From (2.14) we readily obtain that $x_{\infty} \geq 0$ if and only if

$$
s^{2}+\frac{2}{\eta_{k}} s-1 \leq 0
$$

with $\eta_{k}$ given by (2.2). Inequality (2.17) holds for all $0<s \leq s_{k}$ with $s_{k}$ given by (2.1). Thus, to complete (I), it remains to check that $x_{1} \geq 0$ for $k$ odd and $0<s \leq s_{k}$. A computation shows that this condition amounts to

$$
s_{k} \leq \frac{2 b_{k}\left(1-b_{k}\right)}{1-2 b_{k}\left(1-b_{k}\right)} \text { with } b_{k}=\cos \left(\frac{\pi}{k}\right)
$$


However, $s_{k} \leq \frac{1}{2} \eta_{k}$ and $\eta_{k}=4\left(1-b_{k}\right)$ by (2.1-2.2). Substitution yields that (2.18) is true because $b_{k} \leq \frac{1}{2}$ for all $k \geq 3$.

3. Verification of (II). The necessary and sufficient condition for the $k$-gons in (I) not to intersect the line that bisects the angle between the vertical edge $Q_{0}$ and its upper neighboring edge is

$$
\operatorname{Im}\left(v_{N+1}^{j_{1}, \ldots, j_{N}}\left(j_{N}^{*}\right)\right) \leq \frac{1}{2}+\tan \left(\frac{\pi}{k}\right) \operatorname{Re}\left(v_{N+1}^{j_{1}, \ldots, j_{N}}\left(j_{N}^{*}\right)\right) \text { for all indices. }
$$

Indeed, $\pi / k$ is the angle between the horizontal axis and the bisector. Inequality (2.19) says that all the vertices stay below this bisector.

After substituting (2.7-2.8) into (2.9), inserting the expressions for $L, L^{\prime}$, and manipulating to put the real and the imaginary part together, we obtain for condition (II)

$$
\begin{gathered}
(1+s) \cos \left(\frac{\pi}{k}\right)\left\{\sin \left(\frac{\pi}{k}\right)-\sum_{n=1}^{N} s^{n} \sin \left(\pi\left[\sum_{m=1}^{n} \alpha\left(j_{m}\right)-\frac{1}{k}\right]\right)\right\} \\
-s^{N+1} \sin \left(\pi\left[\sum_{m=1}^{N} \alpha\left(j_{m}\right)+\alpha\left(j_{N}^{*}\right)\right]\right) \geq 0 \text { for all indices. }
\end{gathered}
$$

We shall verify that this inequality holds for all $k \geq 3$ and $0<s \leq s_{k}$. For this we shall need to distinguish between the cases $k=3$ and $k \geq 4$.

Case $k \geq 4$. For inequality $(2.20)$ to hold it suffices that

$$
\sin \left(\frac{\pi}{k}\right)-\sum_{n=1}^{N} s^{n}-\frac{s^{N+1}}{(1+s) \cos \left(\frac{\pi}{k}\right)} \geq 0 \text { for all } N \geq 0 .
$$

Since the left hand side is decreasing in $s$ it suffices to check the inequality for $s=s_{k}$. This goes in two steps.

Lemma $1\left(1+s_{k}\right) \cos \left(\frac{\pi}{k}\right) \geq 1$ for $k=4,5, \ldots$

Proof. By (2.1), we obtain (after some arithmetic) that the inequality claimed is equivalent to

$$
\eta_{k}\left[1-2 \cos \left(\frac{\pi}{k}\right)\right]+2 \cos \left(\frac{\pi}{k}\right)-2\left[\cos \left(\frac{\pi}{k}\right)\right]^{2} \leq 0 .
$$

From (2.2) we have

$$
1-\cos \left(\frac{2 \pi}{k}\right) \leq \eta_{k} \leq 4\left[1-\cos \left(\frac{\pi}{k}\right)\right] .
$$

Because $\cos \left(\frac{\pi}{k}\right) \geq \frac{1}{2} \sqrt{2}>\frac{1}{2}$, we see from the lower bound in (2.23) that it suffices to check

$$
\left[1-\cos \left(\frac{2 \pi}{k}\right)\right]\left[1-2 \cos \left(\frac{\pi}{k}\right)\right]+2 \cos \left(\frac{\pi}{k}\right)-2\left[\cos \left(\frac{\pi}{k}\right)\right]^{2} \leq 0,
$$

which is equivalent to $\left(4\left[\cos \left(\frac{\pi}{k}\right)\right]^{2}-2\right)\left(\cos \left(\frac{\pi}{k}\right)-1\right) \leq 0$. But the latter is trivial because $1>\cos \left(\frac{\pi}{k}\right) \geq \frac{1}{2} \sqrt{2}$.

Lemma $2 \sin \left(\frac{\pi}{k}\right) \geq \frac{s_{k}}{1-s_{k}}$ for $k=4,5, \ldots$ 
Proof. From (2.3) we easily check that the inequality claimed holds for $k=4,5$. Therefore let $k=6,7, \ldots$ Again by (2.1), we obtain (after some arithmetic) that the inequality claimed is equivalent to (???)

$$
2 \sin \left(\frac{\pi}{k}\right)\left[1+\sin \left(\frac{\pi}{k}\right)\right] \geq \eta_{k}\left[1+2 \sin \left(\frac{\pi}{k}\right)\right] .
$$

We see from the upper bound in (2.23) that it suffices to check

$$
\sin \left(\frac{\pi}{k}\right) \frac{1+\sin \left(\frac{\pi}{k}\right)}{1+2 \sin \left(\frac{\pi}{k}\right)} \geq 2\left[1-\cos \left(\frac{\pi}{k}\right)\right] .
$$

Since $\sin \left(\frac{\pi}{k}\right) \leq \frac{1}{2}$, it in turn suffices to check that

$$
\frac{3}{4} \sin \left(\frac{\pi}{k}\right) \geq 2\left[1-\cos \left(\frac{\pi}{k}\right)\right] .
$$

But the latter is trivial because the right hand side is $4\left[\sin \left(\frac{\pi}{2 k}\right)\right]^{2}$.

Combine Lemmas 1-2 and use that $s \leq s_{k}$, to obtain (2.21) and hence (2.20) for $k \geq 4$.

Case $k=3$. Return to $(2.20)$. Since $j_{1}=1,2$ and $\alpha(j)=1-\frac{2 j}{3}$, we have that $\sin \left(\pi\left(\alpha\left(j_{1}\right)-\right.\right.$ $\left.\left.\frac{1}{3}\right)\right) \leq 0$. Moreover, $\sum_{m=1}^{n} \alpha\left(j_{m}\right)-\frac{1}{3}$ and $\sum_{m=1}^{N} \alpha\left(j_{m}\right)+\alpha\left(j_{N}^{*}\right)$ are integer multiples of $\frac{1}{3}$. Hence the left hand side of $(2.20)$ is bounded from below by

$$
\sin \left(\frac{\pi}{3}\right)\left[(1+s) \cos \left(\frac{\pi}{3}\right)\left\{1-\sum_{n=2}^{N} s^{n}\right\}-s^{N+1}\right] .
$$

Since $s \leq s_{3}$ and $s_{3}^{2} /\left(1-s_{3}\right)=1$, we have

$$
\sum_{n=2}^{N} s^{n} \leq \sum_{n=2}^{N} s_{3}^{n}=1-\frac{s_{3}^{N+1}}{1-s_{3}} .
$$

Hence we find that (2.28) is bounded from below by

$$
\sin \left(\frac{\pi}{3}\right) s_{3}^{N+1}\left\{\frac{\cos \left(\frac{\pi}{3}\right)}{1-s_{3}}-1\right\}
$$

But this is $\geq 0$ because $\cos \left(\frac{\pi}{3}\right)=\frac{1}{2} \geq 1-s_{3}$, and so we have completed the proof of $(2.20)$ for $k=3$.

The above estimates complete the proof of Theorem 4.

We check that $s_{k}^{2}<1 /(k-1)$ for all $k \geq 3$, which guarantees that our region $D_{s}$ has finite volume for all $0<s \leq s_{k}$ (recall (1.6) and footnote 1). For $k=3,4$ the inequality follows from (2.3). Use (2.1-2.2) to estimate $s_{k} \leq \frac{1}{2} \eta_{k}$ and $\eta_{k} \leq 2(\pi / k)^{2}$, from which the inequality follows for $k \geq 5$.

To conclude this section, note from (2.1-2.2) that

$$
s_{k} \sim\left(\frac{\pi}{k}\right)^{2} \quad(k \rightarrow \infty)
$$

Hence $s_{k}<1 /(k-1)$ for $k$ sufficiently large. One easily checks from $(2.3)$ that $s_{k}>1 /(k-1)$ for $k=3, \ldots, 8$. On the other hand, the estimate $s_{k} \leq(\pi / k)^{2}$ shows that $s_{k}<1 /(k-1)$ for $k=9,10, \ldots$, which isolates the cases for which Theorems 1-3 apply. 


\section{$3 \quad$ Heat content for kites, rectangles and sectors in wedges}

In this section we compute the heat content of certain simple regions. These will later be used as building blocks for our region $D_{s}$. The results will be needed in Section 4 to derive a functional equation for the heat content $E_{D_{s}}(t)$.

\subsection{Kites and rectangles in wedges: Propositions 1-3}

For $0<\gamma<2 \pi$ and $R>0$, we define the wedge and the sector in polar coordinates by

$$
\begin{aligned}
& W_{\gamma}=\{(r, \theta): r>0,0<\theta<\gamma\} \\
& W_{\gamma}(R)=\left\{(r, \theta) \in W_{\gamma}: 0<r<R\right\} .
\end{aligned}
$$

We also define, for $0<\gamma<\pi, R>0$ and $L>0$, the kite (see Fig. 2) and the rectangle in cartesian coordinates by

$$
\begin{aligned}
U_{\gamma}(R) & =\left\{\left(x_{1}, x_{2}\right) \in W_{\gamma}: x_{1}<R, x_{1} \cos \gamma+x_{2} \sin \gamma<R\right\} \\
V(R, L) & =\left\{\left(x_{1}, x_{2}\right):-R<x_{1}<L, 0<x_{2}<R\right\} .
\end{aligned}
$$

Let $u_{W_{\gamma}}$ be the solution of (1.1) for $D=W_{\gamma}$. Abbreviate

$$
d(\gamma)=\frac{\pi}{\gamma} \operatorname{cosec}\left(\frac{\pi^{2}}{\gamma}\right)
$$

which plays a role analogous to (1.4). Our main results in this section are the following three estimates.

Proposition 1 ('kite in non-reflex wedge') For $0<\gamma<\pi$ and $R>0$,

$$
\int_{U_{\gamma}(R)} d x u_{W_{\gamma}}(x ; t)=\frac{4}{\pi^{\frac{1}{2}}} R t^{\frac{1}{2}}-c(\gamma) t+O\left(e^{-\frac{\left[R \sin \left(\frac{\gamma}{2}\right)\right]^{2}}{4 t}}\right) .
$$

Proposition 2 ('kite in reflex wedge') For $0<\gamma<\pi$ and $R>0$,

$$
\int_{U_{\gamma}(R)} d x u_{W_{\pi+\gamma}}(x ; t)=\frac{2}{\pi^{\frac{1}{2}}} R t^{\frac{1}{2}}-[c(\pi+\gamma)+d(\pi+\gamma)] t+O\left(e^{-\frac{\left[R \sin \left(\frac{\gamma}{2}\right)\right]^{2}}{4 t}}\right) .
$$

Proposition 3 ('rectangle in reflex wedge') For $0<\gamma<\pi$ and $L \geq R>0$,

$$
\int_{V(R, L)} d x u_{W_{\pi+\gamma}}(x ; t)=\frac{2}{\pi^{\frac{1}{2}}} L t^{\frac{1}{2}}+d(\pi+\gamma) t+O\left(e^{-\frac{\left[R \sin \left(\frac{\gamma}{2}\right)\right]^{2}}{4 t}}\right) .
$$

The proof of these propositions is organized as follows. In Section 3.2 we compute the integrals over 'sectors in wedges' using the Kontorovich-Lebedev representation of the Dirichlet heat kernel in polar coordinates. In Section 3.3 we derive some probabilistic estimates that will allow us to estimate the contribution of points away from the boundary respectively to compare $u_{D}$ 's for different regions $D$. In Section 3.4 we complete the proof of Propositions $1-3$ using these results. 


\subsection{Sectors in wedges}

The following three lemmas will be needed in Section 3.4 to prove Propositions 1-3. Abbreviate

$$
\eta(R ; t)=\int_{1}^{\infty} \frac{d v}{v^{2}} \int_{0}^{1} \frac{d w w}{\left(1-w^{2}\right)^{\frac{1}{2}}} e^{-\frac{(R v w)^{2}}{4 t}} .
$$

It is easily checked that $\eta(R ; t)=2 t / 3 R^{2}+O\left(t^{2}\right)$ as $t \downarrow 0$.

Lemma 3 ('sector in wedge') For $0<\gamma<\pi$ or $\pi<\gamma<2 \pi$ and $R>0$,

$$
\int_{W_{\gamma}(R)} d x u_{W_{\gamma}}(x ; t)=\frac{4}{\pi^{\frac{1}{2}}} R t^{\frac{1}{2}}[1-\eta(R ; t)]-c(\gamma) t+O\left(e^{-\frac{\left[R \sin \left(\frac{\gamma}{2}\right)\right]^{2}}{4 t}}\right) .
$$

Lemma 4 ('non-reflex sector in reflex wedge') For $0<\gamma<\pi$ and $R>0$,

$$
\int_{W_{\gamma}(R)} d x u_{W_{\pi+\gamma}}(x ; t)=\frac{2}{\pi^{\frac{1}{2}}} R t^{\frac{1}{2}}[1-\eta(R ; t)]-[c(\pi+\gamma)+d(\pi+\gamma)] t+O\left(e^{-\frac{\left[R \sin \left(\frac{\gamma}{2}\right)\right]^{2}}{4 t}}\right) .
$$

Lemma 5 ('half disc in reflex wedge') For $0<\gamma<\pi$ and $R>0$,

$$
\int_{W_{\pi}(R)} d x u_{W_{\pi+\gamma}}(x ; t)=\frac{2}{\pi^{\frac{1}{2}}} R t^{\frac{1}{2}}[1-\eta(R ; t)]+d(\pi+\gamma) t+O\left(e^{-\frac{\left[R \sin \left(\frac{\gamma}{2}\right)\right]^{2}}{4 t}}\right) .
$$

Proof of Lemma 3. Lemma 3 follows from [2] Theorem 2 and Corollary 3.

Proof of Lemma 5. By symmetry,

$$
\int_{W_{\pi}(R)} d x u_{W_{\pi+\gamma}}(x ; t)+\int_{W_{\gamma}(R)} d x u_{W_{\pi+\gamma}}(x ; t)=\int_{W_{\pi+\gamma}(R)} d x u_{W_{\pi+\gamma}}(x ; t) .
$$

Therefore the claim follows directly from Lemmas 3-4.

Proof of Lemma 4. The proof comes in eight parts.

1. Let $-\Delta_{W_{\pi+\gamma}}$ be the Dirichlet Laplacian for $W_{\pi+\gamma}$. Let

$$
p_{W_{\pi+\gamma}}\left(A_{1}, A_{2} ; t\right) \quad\left(A_{1}, A_{2} \in W_{\pi+\gamma}, t \geq 0\right)
$$

denote the heat kernel associated with the parabolic operator $-\Delta_{W_{\pi+\gamma}}+\partial / \partial t$. The Laplace transform of this heat kernel,

$$
G_{W_{\pi+\gamma}}\left(A_{1}, A_{2} ; \lambda\right)=\int_{0}^{\infty} d t p_{W_{\pi+\gamma}}\left(A_{1}, A_{2} ; t\right) e^{-\lambda t} \quad(\lambda>0),
$$

is given by the Kontorovich-Lebedev representation (see [9] p. 44)

$$
\begin{gathered}
G_{W_{\pi+\gamma}}\left(A_{1}, A_{2} ; \lambda\right)=\frac{1}{\pi^{2}} \int_{0}^{\infty} d \zeta K_{i \zeta}\left(\lambda^{\frac{1}{2}} a_{1}\right) K_{i \zeta}\left(\lambda^{\frac{1}{2}} a_{2}\right)\left\{\cosh \left(\left[\pi-\left|\alpha_{1}-\alpha_{2}\right|\right] \zeta\right)\right. \\
\left.-\frac{\sinh (\pi \zeta)}{\sinh ((\pi+\gamma) \zeta)} \cosh \left(\left[(\pi+\gamma)-\alpha_{1}-\alpha_{2}\right] \zeta\right)-\frac{\sinh (\gamma \zeta)}{\sinh ((\pi+\gamma) \zeta)} \cosh \left(\left[\alpha_{1}-\alpha_{2}\right] \zeta\right)\right\},
\end{gathered}
$$


where $A_{1}=\left(a_{1}, \alpha_{1}\right), A_{2}=\left(a_{2}, \alpha_{2}\right)$ are written in polar coordinates and $K_{i \zeta}$ is the modified Bessel function $[9 ; 8.407]$. In the following part we integrate (3.14) in successive steps.

2. First we integrate (3.14) over $\alpha_{1} \in(\gamma, \pi)$ and $\alpha_{2} \in(0, \pi+\gamma)$. This gives, after some straightforward manipulations,

$$
\begin{aligned}
& \int_{\gamma}^{\pi} d \alpha_{1} \int_{0}^{\pi+\gamma} d \alpha_{2} G_{W_{\pi+\gamma}}\left(A_{1}, A_{2} ; \lambda\right)=\frac{2}{\pi^{2}} \int_{0}^{\infty} \frac{d \zeta}{\zeta^{2}} K_{i \zeta}\left(\lambda^{\frac{1}{2}} a_{1}\right) K_{i \zeta}\left(\lambda^{\frac{1}{2}} a_{2}\right) \\
& \quad \times\left\{(\pi-\gamma) \zeta \sinh (\pi \zeta)-\frac{2 \sinh (\pi \zeta)}{\sinh ((\pi+\gamma) \zeta)}(\cosh (\pi \zeta)-\cosh (\gamma \zeta))\right\} .
\end{aligned}
$$

Next, using the identity

$$
\int_{0}^{\infty} d a_{2} a_{2} K_{i \zeta}\left(\lambda^{\frac{1}{2}} a_{2}\right)=\frac{\pi \zeta}{2 \lambda \sinh \left(\frac{\pi \zeta}{2}\right)}
$$

(see $[9 ; 6.561 .16]$ and $[9 ; 8.332 .3]$ ), we can integrate $a_{2}$ times $(3.15)$ over $a_{2} \in(0, \infty)$ and obtain

$$
\begin{aligned}
& \int_{\gamma}^{\pi} d \alpha_{1} \int_{W_{\pi+\gamma}} d A_{2} G_{W_{\pi+\gamma}}\left(A_{1}, A_{2} ; \lambda\right) \\
& \quad=\frac{2}{\pi \lambda} \int_{0}^{\infty} d \zeta K_{i \zeta}\left(\lambda^{\frac{1}{2}} a_{1}\right)\left\{(\pi-\gamma) \cosh \left(\frac{\pi \zeta}{2}\right)-\frac{2}{\zeta} \xi(\gamma ; \zeta)\right\} .
\end{aligned}
$$

Here we have introduced the abbreviation

$$
\xi(\gamma ; \zeta)=\frac{\cosh \left(\frac{\pi}{2} \zeta\right) \sinh \left(\frac{\pi-\gamma}{2} \zeta\right)}{\cosh \left(\frac{\pi+\gamma}{2} \zeta\right)}
$$

Next, using the identity

$$
\int_{0}^{\infty} d \zeta \cosh \left(\frac{\pi \zeta}{2}\right) K_{i \zeta}\left(\lambda^{\frac{1}{2}} a_{1}\right)=\frac{\pi}{2}
$$

(see $[9 ; 6.794 .2]$ ), we can integrate $a_{1}$ times $(3.17)$ over $a_{1} \in(0, R)$ and obtain

$$
\begin{aligned}
& \int_{W_{\pi}(R) \backslash W_{\gamma}(R)} d A_{1} \int_{W_{\pi+\gamma}} d A_{2} G_{W_{\pi+\gamma}}\left(A_{1}, A_{2} ; \lambda\right) \\
& \quad=\frac{(\pi-\gamma) R^{2}}{2 \lambda}-\frac{2}{\lambda^{2}} \int_{0}^{\infty} d \zeta \frac{\xi(\gamma ; \zeta)}{\sinh \left(\frac{\pi \zeta}{2}\right)}+\frac{4}{\pi \lambda} \int_{R}^{\infty} d a_{1} a_{1} \int_{0}^{\infty} \frac{d \zeta}{\zeta} K_{i \zeta}\left(\lambda^{\frac{1}{2}} a_{1}\right) \xi(\gamma ; \zeta) .
\end{aligned}
$$

3. Taking the inverse Laplace transform $\mathcal{L}^{-1}$ of $(3.20)$, we get

$$
\begin{aligned}
& \int_{W_{\pi}(R) \backslash W_{\gamma}(R)} d A_{1} \int_{W_{\pi+\gamma}} d A_{2} p_{W_{\pi+\gamma}}\left(A_{1}, A_{2} ; t\right) \\
& \quad=\frac{(\pi-\gamma) R^{2}}{2}-2 t \int_{0}^{\infty} d \zeta \frac{\xi(\gamma ; \zeta)}{\sinh \left(\frac{\pi \zeta}{2}\right)}+\frac{4}{\pi} \int_{R}^{\infty} d a_{1} a_{1} \int_{0}^{\infty} \frac{d \zeta}{\zeta} \xi(\gamma ; \zeta) \mathcal{L}^{-1}\left\{\frac{1}{\lambda} K_{i \zeta}\left(\lambda^{\frac{1}{2}} a_{1}\right)\right\}(t) .
\end{aligned}
$$

Next, since $p_{W_{\pi+\gamma}}$ is the fundamental solution of the heat equation on $W_{\pi+\gamma}$ with initial temperature 1 and boundary temperature 0 , we have the relation

$$
\int_{W_{\pi+\gamma}} d A_{2} p_{W_{\pi+\gamma}}\left(A_{1}, A_{2} ; t\right)=1-u_{W_{\pi+\gamma}}\left(A_{1} ; t\right) .
$$

Noting that $\left|W_{\pi}(R) \backslash W_{\gamma}(R)\right|=(\pi-\gamma) R^{2} / 2$, we thus arrive at the expression

$$
\begin{aligned}
& \int_{W_{\pi}(R) \backslash W_{\gamma}(R)} d A_{1} u_{W_{\pi+\gamma}}\left(A_{1} ; t\right) \\
& \quad=2 t \int_{0}^{\infty} d \zeta \frac{\xi(\gamma ; \zeta)}{\sinh \left(\frac{\pi \zeta)}{2}\right.}-\frac{4}{\pi} \int_{R}^{\infty} d a_{1} a_{1} \int_{0}^{\infty} \frac{d \zeta}{\zeta} \xi(\gamma ; \zeta) \mathcal{L}^{-1}\left\{\frac{1}{\lambda} K_{i \zeta}\left(\lambda^{\frac{1}{2}} a_{1}\right)\right\}(t) .
\end{aligned}
$$


4. In parts 5-8 below we shall show that the last term in the right hand side of $(3.23)$ is $O\left(\exp \left[-[R \sin (\gamma / 2)]^{2} / 4 t\right]\right)$. But first we complete the proof of Lemma 4 using this fact. Indeed, by symmetry we have

$$
2 \int_{W_{\gamma}(R)} d x u_{W_{\pi+\gamma}}(x ; t)+\int_{W_{\pi}(R) \backslash W_{\gamma}(R)} d x u_{W_{\pi+\gamma}}(x ; t)=\int_{W_{\pi+\gamma}(R)} d x u_{W_{\pi+\gamma}}(x ; t) .
$$

Combining (3.23-3.24) with Lemma 3 and recalling the abbreviation in (3.7), we may now write

$$
\begin{aligned}
& \int_{W_{\gamma}(R)} d x u_{W_{\pi+\gamma}}(x ; t) \\
& \quad=\frac{2}{\pi^{\frac{1}{2}}} R t^{\frac{1}{2}}[1-\eta(R ; t)]-t\left[\frac{1}{2} c(\pi+\gamma)+\int_{0}^{\infty} d \zeta \frac{\xi(\gamma ; \zeta)}{\sinh \left(\frac{\pi \zeta}{2}\right)}\right]+O\left(e^{-\frac{\left[R \sin \left(\frac{\gamma}{2}\right)\right]^{2}}{4 t}}\right) .
\end{aligned}
$$

Next, recalling (1.4) and (3.18), we write

$$
\begin{aligned}
\int_{0}^{\infty} d \zeta \frac{\xi(\gamma ; \zeta)}{\sinh \left(\frac{\pi \zeta}{2}\right)}-\frac{1}{2} c(\pi+\gamma) & =2 \int_{0}^{\infty} d \zeta\left\{\frac{\cosh (\pi \zeta)}{\sinh (\pi \zeta)} \frac{\sinh ((\pi-\gamma) \zeta)}{\cosh ((\pi+\gamma) \zeta)}+\frac{\sinh (\gamma \zeta)}{\sinh (\pi \zeta) \cosh ((\pi+\gamma) \zeta)}\right\} \\
& =2 \int_{0}^{\infty} d \zeta \frac{\cosh ((\pi-\gamma) \zeta)}{\cosh ((\pi+\gamma) \zeta)} \\
& =\frac{\pi}{\pi+\gamma} \operatorname{cosec}\left(\frac{\pi^{2}}{\pi+\gamma}\right) \\
& =d(\pi+\gamma),
\end{aligned}
$$

where the third equality uses $[9 ; 3.524 .6]$. The claim in Lemma 4 follows by combining $(3.25)$ and (3.26).

5. To complete the proof of Lemma 4, we estimate the last term in the right hand side of (3.23). For this we need to distinguish three regimes for $\gamma$ (see parts 6- 8 below). The key relations are

$$
\begin{array}{ll}
K_{i \zeta}\left(\lambda^{\frac{1}{2}} a\right) & =\int_{0}^{\infty} d u \cos (\zeta u) e^{-\lambda^{\frac{1}{2}} a \cosh u} \\
\mathcal{L}^{-1}\left\{\frac{1}{\lambda} K_{i \zeta}\left(\lambda^{\frac{1}{2}} a\right)\right\}(t) & =\int_{0}^{\infty} d u \cos (\zeta u) \operatorname{Erfc}\left(\frac{a \cosh u}{2 t^{\frac{1}{2}}}\right),
\end{array}
$$

which follow from $[9 ; 3.547 .4]$ respectively $[8 ; 5.6 .3]$. Here Erfc is the complementary error function defined by $\operatorname{Erfc}(x)=\frac{2}{\pi^{1 / 2}} \int_{x}^{\infty} \exp \left(-x^{2}\right) d x$.

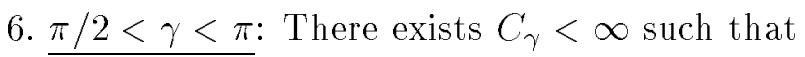

$$
\left|\frac{1}{\zeta} \xi(\gamma ; \zeta)\right| \leq C_{\gamma} e^{-\left(\gamma-\frac{\pi}{2}\right) \zeta} \quad(\zeta>0) .
$$


From (3.27-3.28) we obtain

$$
\begin{aligned}
\mid \text { last term in }(3.23) \mid & \leq \frac{4}{\pi} C_{\gamma} \int_{0}^{\infty} d u \int_{R}^{\infty} d a a \operatorname{Erfc}\left(\frac{a \cosh u}{2 t^{\frac{1}{2}}}\right)\left[\int_{0}^{\infty} d \zeta|\cos (\zeta u)| e^{-\left(\gamma-\frac{\pi}{2}\right) \zeta}\right] \\
& \leq \frac{8 C_{\gamma}}{\pi(2 \gamma-\pi)} \int_{0}^{\infty} d u \int_{R}^{\infty} d a a \operatorname{Erfc}\left(\frac{a \cosh u}{2 t^{\frac{1}{2}}}\right) \\
& =\frac{8 C_{\gamma}}{\pi^{\frac{3}{2}}(2 \gamma-\pi) t^{\frac{1}{2}}} \int_{R}^{\infty} d a a^{2} \int_{0}^{\infty} d u u(\sinh u) e^{-\frac{(a \cosh u)^{2}}{4 t}} \\
& \leq \frac{8 C_{\gamma}}{\pi^{\frac{3}{2}}(2 \gamma-\pi) t^{\frac{1}{2}}} \int_{R}^{\infty} d a a^{2} \int_{0}^{\infty} d u u(\sinh u)(\cosh u) e^{-\frac{(a \cosh u)^{2}}{4 t}} \\
& =\frac{16 C_{\gamma} t^{\frac{1}{2}}}{\pi^{\frac{3}{2}}(2 \gamma-\pi)} \int_{R}^{\infty} d a e^{-\frac{a^{2}}{4 t}} \\
& =O\left(e^{-\frac{R^{2}}{4 t}}\right) .
\end{aligned}
$$

7. $\gamma=\pi / 2$ : Integration by parts with respect to $u$ gives the equality

$$
\mathcal{L}^{-1}\left\{\frac{1}{\lambda} K_{i \zeta}\left(\lambda^{\frac{1}{2}} a\right)\right\}(t)=\frac{1}{\zeta} \int_{0}^{\infty} d u \sin (\zeta u)\left[\frac{2}{\pi^{\frac{1}{2}}} \frac{a \sinh u}{2 t^{\frac{1}{2}}} e^{-\frac{(a \cosh u)^{2}}{4 t}}\right] .
$$

Since $|\sin (\zeta u)| \leq(\zeta u)^{\frac{1}{2}}$ for all $\zeta, u \geq 0$, we obtain that

$$
\mid \text { last term in }(3.23) \mid \leq \frac{4}{\pi^{\frac{3}{2}} t^{\frac{1}{2}}} \int_{R}^{\infty} d a a^{2} \int_{0}^{\infty} d u u^{\frac{1}{2}}(\sinh u) e^{-\frac{(a \cosh u)^{2}}{4 t}} \int_{0}^{\infty} \frac{d \zeta}{\zeta^{\frac{3}{2}}} \xi\left(\frac{\pi}{2} ; \zeta\right) .
$$

In the last integral, $\xi\left(\frac{\pi}{2} ; \zeta\right) \sim \frac{\pi \zeta}{4}(\zeta \downarrow 0)$ and $\xi\left(\frac{\pi}{2} ; \zeta\right) \rightarrow \frac{1}{2}(\zeta \rightarrow \infty)$. Hence this integral converges and (3.31) is bounded by

$$
\begin{aligned}
& \frac{C}{t^{\frac{1}{2}}} \int_{R}^{\infty} d a a^{2} \int_{0}^{\infty} d u u^{\frac{1}{2}}(\sinh u) e^{-\frac{(a \cosh u)^{2}}{4 t}} \\
& \quad \leq \frac{C}{t^{\frac{1}{2}}} \int_{R}^{\infty} d a a^{2} \int_{0}^{\infty} d u(\sinh u)(\cosh u) e^{-\frac{(a \cosh u)^{2}}{4 t}} \\
& \quad=O\left(e^{-\frac{R^{2}}{4 t}}\right)
\end{aligned}
$$

for some $C<\infty$.

8. $0<\gamma<\pi / 2$ : Write

$$
\xi(\gamma ; \zeta)=\sinh \left(\left(\frac{\pi}{2}-\gamma\right) \zeta\right)+\hat{\xi}(\gamma ; \zeta),
$$

where we introduce

$$
\hat{\xi}(\gamma ; \zeta)=\frac{\cosh (\gamma \zeta) \sinh \left(\frac{\gamma}{2} \zeta\right)}{\cosh \left(\frac{\pi+\gamma}{2} \zeta\right)}
$$


There exists $D_{\gamma}<\infty$ such that

$$
\left|\frac{1}{\zeta} \hat{\xi}(\gamma ; \zeta)\right| \leq D_{\gamma} e^{-\left(\frac{\pi}{2}-\gamma\right) \zeta} \quad(\zeta>0) .
$$

Therefore, using (3.27) and estimates similar as those in (3.29), we obtain

$$
\begin{aligned}
& \left|\frac{4}{\pi} \int_{R}^{\infty} d a a \int_{0}^{\infty} \frac{d \zeta}{\zeta} \hat{\xi}(\gamma ; \zeta) \mathcal{L}^{-1}\left\{\frac{1}{\lambda} K_{i \zeta}\left(\lambda^{\frac{1}{2}} a\right)\right\}(t)\right| \\
& \quad \leq \frac{4 D_{\gamma}}{\pi} \int_{0}^{\infty} d u \int_{R}^{\infty} d a a \operatorname{Erfc}\left(\frac{a \cosh u}{2 t^{\frac{1}{2}}}\right)\left[\int_{0}^{\infty} d \zeta|\cos (\zeta u)| e^{-\left(\frac{\pi}{2}-\gamma\right) \zeta}\right] \\
& \quad \leq \frac{8 D_{\gamma}}{\pi(\pi-2 \gamma)} \int_{0}^{\infty} d u \int_{R}^{\infty} d a a \operatorname{Erfc}\left(\frac{a \cosh u}{2 t^{\frac{1}{2}}}\right) \\
& \quad=O\left(e^{-\frac{R^{2}}{4 t}}\right)
\end{aligned}
$$

On the other hand, by (3.27),

$$
\begin{aligned}
& \left.\frac{4}{\pi} \int_{R}^{\infty} d a a \int_{0}^{\infty} \frac{d \zeta}{\zeta} \sinh \left(\left(\frac{\pi}{2}-\gamma\right) \zeta\right)\right) \mathcal{L}^{-1}\left\{\frac{1}{\lambda} K_{i \zeta}\left(\lambda^{\frac{1}{2}} a\right)\right\}(t) \\
& \quad=\frac{4}{\pi} \int_{R}^{\infty} d a a \mathcal{L}^{-1}\left\{\frac{1}{\lambda} \int_{0}^{\frac{\pi}{2}-\gamma} d \alpha\left[\int_{0}^{\infty} d \zeta \cosh (\alpha \zeta) K_{i \zeta}\left(\lambda^{\frac{1}{2}} a\right)\right]\right\}(t) \\
& \quad=2 \int_{R}^{\infty} d a a \mathcal{L}^{-1}\left\{\frac{1}{\lambda} \int_{0}^{\frac{\pi}{2}-\gamma} d \alpha e^{-\lambda^{\frac{1}{2}} a \cos \alpha}\right\}(t) \\
& \quad=2 \int_{R}^{\infty} d a a \int_{0}^{\frac{\pi}{2}-\gamma} d \alpha \operatorname{Erfc}\left(\frac{a \cos \alpha}{2 t^{\frac{1}{2}}}\right) \\
& \quad \leq(\pi-2 \gamma) \int_{R}^{\infty} d a a \operatorname{Erfc}\left(\frac{a \cos \left(\frac{\pi}{2}-\gamma\right)}{2 t^{\frac{1}{2}}}\right) \\
& \quad=O\left(e^{-\frac{\left[R \sin \left(\frac{\gamma}{2}\right)\right]^{2}}{4 t}}\right),
\end{aligned}
$$

where the second equality uses $[9 ; 6.795 .1]$ and the third equality $[8 ; 5.6 .3]$. Combining (3.36) and (3.37), we get the remainder estimate as claimed.

\subsection{Three comparison lemmas}

Lemmas $6-8$ below are technical estimates that will be needed later on to compare the heat content of different regions.

Let $(B(t): t \geq 0)$ be standard Brownian motion on $\mathbb{R}^{2}$, i.e., the process with generator $-\Delta+\partial / \partial t$. Write $P_{x}$ to denote its probability law given $B(0)=x$. For $A \subset \mathbb{R}^{2}$ open, define the first exit time

$$
T_{A}=\inf \{t \geq 0: B(t) \notin A\} \quad(B(0) \in A) .
$$

Then for $D$ open the solution $u_{D}$ of (1.1) has the well-known representation

$$
u_{D}(x ; t)=P_{x}\left[T_{D} \leq t\right] \quad(x \in D) .
$$

The following lemma is known as 'Kac's principle of not feeling the boundary' [12]. 
Lemma 6 For $x \in D$ and $t>0$,

$$
u_{D}(x ; t) \leq 2 e^{-\frac{d^{2}(x, \partial D)}{4 t}}
$$

where $d(x, \partial D)=\min \{|x-y|: y \in \partial D\}$.

Proof. By Levy's maximal inequality ([11] Theorem 3.6.5)

$$
\begin{aligned}
P_{x}\left[T_{D} \leq t\right] & \leq 2 P_{0}[|B(t)| \geq d(x, \partial D)] \\
& =\frac{1}{2 \pi t} \int_{\{|y|>d(x, \partial D)\}} d y e^{-\frac{|y|^{2}}{4 t}} \\
& =2 e^{-\frac{d^{2}(x, \partial D)}{4 t}} .
\end{aligned}
$$

Next we formulate two lemmas that will allow us to compare solutions of (1.1) for different regions. These will serve us later when we approximate parts of $D_{s}$ by the simpler regions studied in Propositions 1-3.

Lemma 7 Let $u_{D}$ and $u_{F}$ be the solutions of (1.1) for two open sets $D$ resp. $F$ in $\mathbb{R}^{2}$. Suppose that $E \subset(D \cap F)$, and that $E$ is measurable. Then

$$
\left|\int_{E} d x u_{D}(x ; t)-\int_{E} d x u_{F}(x ; t)\right| \leq 2|E| e^{-\frac{\epsilon^{2}}{4 t}}
$$

with

$$
\epsilon=\inf \{|x-y|: x \in E, y \in \overline{D \triangle F}\},
$$

where $D \triangle F$ is the symmetric difference of $D$ and $F$.

Proof. Write, using (3.39),

$$
\begin{aligned}
u_{D}(x ; t) & \leq u_{D \cap F}(x ; t)=P_{x}\left[T_{D \cap F} \leq t\right] \\
& =P_{x}\left[T_{D \cap F} \leq t, T_{F} \leq t\right]+P_{x}\left[T_{D \cap F} \leq t, T_{F}>t\right] \\
& \leq u_{F}(x ; t)+P_{x}\left[T_{D \cap F} \leq t, T_{F}>t\right] \quad(x \in D \cap F) .
\end{aligned}
$$

For $A \subset \mathbb{R}^{2}$ closed, define the first entrance time

$$
\tau_{A}=\inf \{t \geq 0: B(t) \in A\} \quad(B(0) \notin A) .
$$

Note that

$$
T_{D}=\tau_{\partial D}=\tau_{D^{c}}
$$


It therefore follows from Lemma 6 and (3.43) that

$$
\begin{aligned}
P_{x}\left[T_{D \cap F} \leq t, T_{F}>t\right] & =P_{x}\left[\tau_{(D \cap F)^{c}} \leq t, \tau_{F^{c}}>t\right] \\
& =P_{x}\left[\tau_{D \triangle F} \leq t, \tau_{F^{c}}>t\right] \\
& \leq P_{x}\left[\tau_{D \triangle F} \leq t\right] \\
& =P_{x}\left[T_{(D \triangle F)^{c}} \leq t\right] \\
& \leq P_{x}\left[T(\overline{D \triangle F})^{c} \leq t\right] \\
& \leq 2 e^{-\frac{\epsilon^{2}}{4 t}} \quad(x \in D \cap F) .
\end{aligned}
$$

Combining (3.44), (3.47) and reversing the role of $D$ and $F$, we get

$$
\left|u_{D}(x ; t)-u_{F}(x ; t)\right| \leq 2 e^{-\frac{\epsilon^{2}}{4 t}} \quad(x \in D \cap F) .
$$

Integrate over $x \in E$ to get the claim.

Lemma 8 Let $D, F$ and $G$ be open sets in $\mathbb{R}^{2}$ such that $G \subset(D \cap F)$. Suppose that $E$ is a bounded measurable subset of $G$. Then

$$
\left|\int_{E} d x u_{D}(x ; t)-\int_{E} d x u_{F}(x ; t)\right| \leq 2|E| e^{-\frac{\epsilon^{2}}{4 t}}
$$

with

$$
\epsilon=\inf \{|x-y|: x \in E, y \in \overline{(D \cup F) \cap \partial G}\}
$$

Proof. Write

$$
\begin{aligned}
u_{D}(x ; t) & =P_{x}\left[T_{D} \leq t\right] \\
& \leq P_{x}\left[T_{F} \leq t\right]+P_{x}\left[T_{D} \leq t, T_{F}>t\right] \\
& \leq u_{F}(x ; t)+P_{x}\left[T_{G} \leq t, T_{F}>t\right]
\end{aligned}
$$

and

$$
\begin{aligned}
P_{x}\left[\tau_{\partial G} \leq t, T_{F}>t\right] & \leq P_{x}[\tau \overline{\partial G \cap F} \leq t] \\
& \leq P_{x}[\tau \overline{\partial G \cap(D \cup F)} \leq t] \quad(x \in G) .
\end{aligned}
$$

By symmetry, the same inequality holds with $D$ and $F$ interchanged. Hence we obtain (3.48) with $\epsilon$ given by (3.50). Integration over $x \in E$ yields (3.49) (recall (3.39) and (3.46)). 


\subsection{Proof of Propositions 1-3}

With the help of Lemmas 3-7 we can now prove Propositions 1-3.

Proof of Proposition 1. The proof comes in four parts.

1. For $0<\gamma<\pi$ and $R>0$, define (see Fig. 3)

$$
\begin{aligned}
& C_{\gamma}^{+}(R)=\left\{(r, \theta) \in U_{\gamma}(R) \backslash W_{\gamma}(R): 0<\theta<\frac{\gamma}{2}\right\} \\
& C_{\gamma}^{-}(R)=\left\{(r, \theta) \in U_{\gamma}(R) \backslash W_{\gamma}(R): \frac{\gamma}{2}<\theta<\gamma\right\}
\end{aligned}
$$

By symmetry,

$$
\int_{C_{\gamma}^{+}(R)} d x u_{W_{\gamma}}(x ; t)=\int_{C_{\gamma}^{-}(R)} d x u_{W_{\gamma}}(x ; t) .
$$

Hence the integral in Proposition 1 equals

$$
\int_{U_{\gamma}(R)} d x u_{W_{\gamma}}(x ; t)=\int_{W_{\gamma}(R)} d x u_{W_{\gamma}}(x ; t)+2 \int_{C_{\gamma}^{+}(R)} d x u_{W_{\gamma}}(x ; t) .
$$

The integral over $W_{\gamma}(R)$ we know from Lemma 3. Thus it remains to compute the integral over $C_{\gamma}^{+}(R)$.

2. To that end, we use Lemma 7 to approximate $u_{W_{\gamma}}(x ; t)$ by $u_{W_{\pi}}(x ; t)$ for $x \in C_{\gamma}^{+}(R)$, as follows. Choose $D=W_{\gamma}, F=W_{\pi}$ and $E=C_{\gamma}^{+}(R)$. Then $\epsilon$ in (3.43) becomes

$$
\epsilon=R \sin \left(\frac{\gamma}{2}\right)
$$

so

$$
\int_{C_{\gamma}^{+}(R)} d x u_{W_{\gamma}}(x ; t)=\int_{C_{\gamma}^{+}(R)} d x u_{W_{\pi}}(x ; t)+O\left(e^{-\frac{\left[R \sin \left(\frac{\gamma}{2}\right)\right]^{2}}{4 t}}\right) .
$$

3. Next, for $x=\left(x_{1}, x_{2}\right) \in W_{\pi}$ we have, by (1.1),

$$
u_{W_{\pi}}(x ; t)=\frac{1}{(\pi t)^{\frac{1}{2}}} \int_{x_{2}}^{\infty} d q e^{-\frac{q^{2}}{4 t}} .
$$

Hence

$$
\begin{aligned}
& \int_{C_{\gamma}^{+}(R)} d x u_{W_{\pi}}(x ; t) \\
& \quad=\frac{1}{(\pi t)^{\frac{1}{2}}} \int_{0}^{R \sin \left(\frac{\gamma}{2}\right)} d x_{2} \int_{\left(R^{2}-x_{2}^{2}\right)^{\frac{1}{2}}}^{R} d x_{1} \int_{x_{2}}^{\infty} d q e^{-\frac{q^{2}}{4 t}}+O\left(e^{-\frac{\left[R \sin \left(\frac{\gamma}{2}\right)\right]^{2}}{4 t}}\right) \\
& =\frac{1}{(\pi t)^{\frac{1}{2}}} \int_{0}^{R \sin \left(\frac{\gamma}{2}\right)} d x_{2}\left\{R-\left(R^{2}-x_{2}^{2}\right)^{\frac{1}{2}}\right\} \int_{x_{2}}^{\infty} d q e^{-\frac{q^{2}}{4 t}}+O\left(e^{-\frac{\left[R \sin \left(\frac{\gamma}{2}\right)\right]^{2}}{4 t}}\right) .
\end{aligned}
$$

Combining Lemma $3,(3.55),(3.57)$ and (3.59), we thus find that

$$
\int_{U \gamma(R)} d x u_{W_{\gamma}}(x ; t)=\frac{4}{\pi^{\frac{1}{2}}} R t^{\frac{1}{2}}[1-\eta(R ; t)]-c(\gamma) t+O\left(e^{-\frac{\left[R \sin \left(\frac{\gamma}{2}\right)\right]^{2}}{4 t}}\right)+2 \text { r.h.s. }(3.59) .
$$


4. Now, by changing variable and integrating by parts, we get

$$
\begin{aligned}
& \int_{0}^{R \sin \left(\frac{\gamma}{2}\right)} d x_{2}\left\{R-\left(R^{2}-x_{2}^{2}\right)^{\frac{1}{2}}\right\} \int_{x_{2}}^{\infty} d q e^{-\frac{q^{2}}{4 t}} \\
& \quad=\int_{1}^{\infty} d v\left(-\frac{2 R t}{v^{2}}\right) \int_{0}^{\sin \left(\frac{\gamma}{2}\right)}\left\{1-\left(1-w^{2}\right)^{\frac{1}{2}}\right\} \frac{d}{d w}\left(e^{-\frac{(v w R)^{2}}{4 t}}\right) \\
& \quad=-2 R t \int_{1}^{\infty} \frac{d v}{v^{2}}\left\{\left(1-\cos \left(\frac{\gamma}{2}\right)\right) e^{-\frac{\left[v R \sin \left(\frac{\gamma}{2}\right)\right]^{2}}{4 t}}-\int_{0}^{\sin \left(\frac{\gamma}{2}\right)} \frac{d w w}{\left(1-w^{2}\right)^{\frac{1}{2}}} e^{-\frac{(v w R)^{2}}{4 t}}\right\} .
\end{aligned}
$$

This last integral is a truncated form of (3.7) and we therefore get

$$
\begin{aligned}
& -\frac{4}{\pi^{\frac{1}{2}}} R t^{\frac{1}{2}} \eta(R ; t)+2 \frac{1}{(\pi t)^{\frac{1}{2}}} \int_{0}^{R \sin \left(\frac{\gamma}{2}\right)} d x_{2}\left\{R-\left(R^{2}-x_{2}^{2}\right)^{\frac{1}{2}}\right\} \int_{x_{2}}^{\infty} d q e^{-\frac{q^{2}}{4 t}} \\
& =-\frac{4}{\pi^{\frac{1}{2}}} R t^{\frac{1}{2}} \int_{1}^{\infty} \frac{d v}{v^{2}}\left\{\left(1-\cos \left(\frac{\gamma}{2}\right)\right) e^{\frac{-\left[v R \sin \left(\frac{\gamma}{2}\right)\right]^{2}}{4 t}}+\int_{\sin \left(\frac{\gamma}{2}\right)}^{1} \frac{d w w}{\left(1-w^{2}\right)^{\frac{1}{2}}} e^{-\frac{(v w R)^{2}}{4 t}}\right\} \\
& =O\left(e^{-\frac{\left[R \sin \left(\frac{\gamma}{2}\right)\right]^{2}}{4 t}}\right) .
\end{aligned}
$$

Note that a cancellation occurs here of terms that are polynomial in $t$ as $t \downarrow 0$. Proposition 1 follows by combining (3.59-3.62).

Proof of Proposition 2. The proof comes in four parts.

1. The integral in Proposition 2 equals

$$
\begin{aligned}
& \int_{U_{\gamma}(R)} d x u_{W_{\pi+\gamma}}(x ; t) \\
& \quad=\int_{W_{\gamma}(R)} d x u_{W_{\pi+\gamma}}(x ; t)+\int_{C_{\gamma}^{+}(R)} d x u_{W_{\pi+\gamma}}(x ; t)+\int_{C_{\gamma}^{-}(R)} d x u_{W_{\pi+\gamma}}(x ; t) .
\end{aligned}
$$

The integral over $W_{\gamma}(R)$ we know from Lemma 4 .

2. The distance between $C_{\gamma}^{-}(R)$ and $\partial W_{\pi+\gamma}$ is $R \sin (\gamma / 2)$. Hence it follows from Lemma 6 that

$$
\int_{C_{\gamma}^{-}(R)} d x u_{W_{\pi+\gamma}}(x ; t) \leq 2\left|C_{\gamma}^{-}(R)\right| e^{-\frac{\left[R \sin \left(\frac{\gamma}{2}\right)\right]^{2}}{4 t}} .
$$

3. To estimate the contribution from $C_{\gamma}^{+}(R)$ we again use Lemma 7 , this time with $D=W_{\pi+\gamma}$, $F=W_{\pi}$ and $E=C_{\gamma}^{+}(R)$. Then in $(3.43) \epsilon=R$ if $0<\gamma / \pi / 2$ and $\epsilon=R \sin \gamma$ if $\pi / 2<\gamma<\pi$. Hence $\epsilon \geq R \sin (\gamma / 2)$ in either case, and so

$$
\int_{C_{\gamma}^{+}(R)} d x u_{W_{\pi+\gamma}}(x ; t)=\int_{C_{\gamma}^{+}(R)} d x u_{W_{\pi}}(x ; t)+O\left(e^{-\frac{\left[R \sin \left(\frac{\gamma}{2}\right)\right]^{2}}{4 t}}\right) .
$$

4. It now follows from Lemma $4,(3.59)$ and (3.64) that

$$
\int_{U_{\gamma}(R)} d x u_{W_{\pi+\gamma}}(x ; t)=\frac{2}{\pi^{\frac{1}{2}}} R t^{\frac{1}{2}}[1-\eta(R ; t)]-[c(\pi+\gamma)+d(\pi+\gamma)] t+\text { r.h.s.(3.59). }
$$

Again there is a cancellation, as in (3.62), leading to the same error estimate. 
Proof of Proposition 3. The proof comes in four parts.

1. For $L \geq R>0$, define (see Fig. 4)

$$
\begin{aligned}
& D^{+}(R, L)=\left\{\left(x_{1}, x_{2}\right) \in V(R, L) \backslash W_{\pi}(R): 0<x_{1}<L\right\} \\
& D^{-}(R, L)=\left\{\left(x_{1}, x_{2}\right) \in V(R, L) \backslash W_{\pi}(R):-R<x_{1}<0\right\} .
\end{aligned}
$$

The integral in Proposition 3 equals

$$
\begin{aligned}
& \int_{V(R, L)} d x u_{W_{\pi+\gamma}}(x ; t) \\
& \quad=\int_{W_{\pi}(R)} d x u_{W_{\pi+\gamma}}(x ; t)+\int_{D^{+}(R, L)} d x u_{W_{\pi+\gamma}}(x ; t)+\int_{D^{-}(R, L)} d x u_{W_{\pi+\gamma}}(x ; t) .
\end{aligned}
$$

The integral over $W_{\pi}(R)$ we know from Lemma 5 .

2. Pick $0<\gamma<\pi$. The distance between $D^{-}(R, L)$ and $\partial W_{\pi+\gamma}$ is $R \sin \gamma$ if $0<\gamma<\pi / 2$ and is $R$ if $\pi / 2<\gamma<\pi$. Hence $\epsilon \geq R \sin (\gamma / 2)$ in either case, and

$$
\int_{D^{-}(R, L)} d x u_{W_{\pi+\gamma}}(x ; t) \leq 2\left|D^{-}(R, L)\right| e^{-\frac{\left[R \sin \left(\frac{\gamma}{2}\right)\right]^{2}}{4 t}}
$$

by Lemma 6 . 3. To estimate the contribution from $D^{+}(R, L)$, we pick $D=W_{\pi+\gamma}, F=W_{\pi}$, $E=D^{+}(R, L)$ and $G=\left\{x \in D: d\left(x, D^{+}(R, L)\right)<R\right\}$ in Lemma 8. Then $\epsilon=R$ in (3.50). We obtain via (3.58) that

$$
\begin{aligned}
& \int_{D^{+}(R, L)} d x u_{W_{\pi+\gamma}}(x ; t)=\int_{D^{+}(R, L)} d x u_{W_{\pi}}(x ; t)+O\left(e^{-\frac{R^{2}}{4 t}}\right) \\
& \quad=\frac{1}{(\pi t)^{\frac{1}{2}}} \int_{0}^{R} d x_{2}\left(L-\left(R^{2}-x_{2}^{2}\right)^{\frac{1}{2}}\right) \int_{x_{2}}^{\infty} d q e^{-\frac{q^{2}}{4 t}}+O\left(e^{-\frac{R^{2}}{4 t}}\right) \\
& \quad=\frac{1}{(\pi t)^{\frac{1}{2}}} \int_{0}^{R} d x_{2}\left(R-\left(R^{2}-x_{2}^{2}\right)^{\frac{1}{2}}\right) \int_{x_{2}}^{\infty} d q e^{-\frac{q^{2}}{4 t}}+\frac{2}{\pi^{\frac{1}{2}}}(L-R) t^{\frac{1}{2}}+O\left(e^{-\frac{R^{2}}{4 t}}\right) .
\end{aligned}
$$

4. It now follows from Lemma $5,(3.69)$ and (3.70) that

$$
\int_{V(R, L)} d x u_{W_{\pi+\gamma}}(x ; t)=\frac{2}{\pi^{\frac{1}{2}}} L t^{\frac{1}{2}}-\frac{2}{\pi^{\frac{1}{2}}} R t^{\frac{1}{2}} \eta(R ; t)+d(\pi+\gamma) t+\text { r.h.s.(3.59). }
$$

Once again there is a cancellation between the last two terms, so that the error estimate is the same as before.

\section{Functional equation: Proof of Theorems 1-3}

Propositions 1-3 are the key to Theorems 1-3. The proof will rely on the approximation Lemmas 7-8. In Section 4.1 we compute the heat content of the basic $k$-gon $Q_{0}$ respectively one of the $k$-gons $Q_{1,1}$ attached to it. In Section 4.2 we use this information to derive a functional equation for the heat content $E_{D_{s}}(t)$ of our region $D_{s}$. From this functional equation we shall be able to draw out the expansions in Theorems 1-3. In Section 5 we shall study the periodic function $p_{s}$ that appears in these expansions and prove the claims that were made about it in Section 1. 


\subsection{Heat content of two building blocks of $D_{s}$}

We first estimate the contribution from the basic $k$-gon $Q_{0}$.

Lemma 9 Fix $k \geq 3$. If $0<s \leq s_{k}$, then

$$
\int_{Q_{0}} d x u_{D_{s}}(x ; t)=\frac{2}{\pi^{\frac{1}{2}}} k(1-s) t^{\frac{1}{2}}+k\left[-c\left(\gamma_{k}\right)+2 d\left(\pi+\gamma_{k}\right)\right] t+O\left(e^{-\frac{\delta_{s}}{t}}\right),
$$

where $\gamma_{k}, c(\gamma), d(\gamma)$ are defined in defined in (1.13), (1.4) respectively (3.3), and

$$
\delta_{s}= \begin{cases}\frac{s^{2}}{64}(3-\sqrt{5})(2-\sqrt{3}) & (k=3) \\ \frac{s^{2}}{8}(3-2 \sqrt{2}) & (k=4) \\ \frac{s^{2}}{16} \sin ^{2}\left(\frac{\pi}{k}\right) & (k \geq 5) .\end{cases}
$$

Proof. Let

$$
\begin{aligned}
& R_{s}=\min \left\{\frac{s}{2}, \frac{1-s}{2\left(1+\cot \left(\frac{\gamma_{k}}{2}\right)\right)}\right\} \\
& L_{s}=\frac{1-s}{2}-R_{s} \cot \left(\frac{\gamma_{k}}{2}\right) .
\end{aligned}
$$

This choice guarantees that the following three conditions are met (see Fig. 5):

$$
\begin{aligned}
\frac{1-s}{2} & =L_{s}+R_{s} \cot \left(\frac{\gamma_{k}}{2}\right) \\
R_{s} & \leq \frac{s}{2} \\
R_{s} & \leq L_{s} .
\end{aligned}
$$

Partition $Q_{0}$ into the following sets (see Fig. 5):

(i) $k$ kites $U_{\gamma_{k}}\left(R_{s} \cot \left(\frac{\gamma_{k}}{2}\right)\right)$.

(ii) $2 k$ rectangles $V\left(R_{s}, L_{s}\right)$.

(iii) The interior of $Q_{0}$ minus (i) and (ii): an open polygon with distance $\epsilon_{0}$ to $\partial D_{s}$, where

$$
\epsilon_{0}= \begin{cases}R_{s} & \left(k \geq 4 \text { or } k=3, R_{s}=\frac{s}{2}\right) \\ \frac{1}{2} \sqrt{3} R_{s} & \left(k=3, R_{s}=L_{s}\right) .\end{cases}
$$

(iv) The remainder, which has measure zero.

1. By Lemma 6 the contribution of (iii) is $O\left(\exp \left(-\epsilon_{0}^{2} / 8 t\right)\right)$.

2. In order to estimate the contribution of (i) we may again apply Lemma 7 , this time with $D=D_{s}, F=W_{\gamma_{k}}$ and $E=U_{\gamma_{k}}\left(R_{s} \cot \left(\frac{\gamma_{k}}{2}\right)\right)$. Then $\epsilon$ in (3.43) becomes

$$
\epsilon_{1}=L_{s}
$$

Together with Proposition 1 we therefore get a contribution

$$
k\left[\frac{4}{\pi^{\frac{1}{2}}} R t^{\frac{1}{2}}-c\left(\gamma_{k}\right) t\right]+O\left(e^{-\frac{R_{s}^{2} \cos ^{2}\left(\frac{\gamma_{k}}{2}\right)}{4 t}}\right)+O\left(e^{-\frac{\epsilon_{1}^{2}}{4 t}}\right)
$$


where we use that $\left[R_{s} \cot \left(\frac{\gamma_{k}}{2}\right) \sin \left(\frac{\gamma_{k}}{2}\right]^{2}=R_{s}^{2} \cos ^{2}\left(\frac{\gamma_{k}}{2}\right)\right.$.

3. The contribution of (ii) can be estimated via Lemma 8 after picking $D=D_{s}, F=W_{\pi+\gamma_{k}}$, $E=V\left(R_{s}, L_{s}\right)$ and

$$
G=\left\{x \in D_{s}: \operatorname{dist}\left(x, V\left(R_{s}, L_{s}\right)\right)<\epsilon_{2}\right\},
$$

where

$$
\epsilon_{2}= \begin{cases}\min \left\{R_{s}, \frac{s(1-s)}{2} \sin \gamma_{k}\right\} & (k=3) \\ \min \left\{R_{s} \cot \left(\frac{\gamma_{k}}{2}\right), \frac{s(1-s)}{2} \sin \gamma_{k}\right\} & (k \geq 4) .\end{cases}
$$

This choice guarantees that $G \in W_{\pi+\gamma_{k}}$ (see Fig. 5). Then $\epsilon$ in (3.50) becomes

$$
\epsilon=\epsilon_{2} \text {. }
$$

Together with Proposition 3 we therefore get a contribution

$$
2 k\left[\frac{2}{\pi^{\frac{1}{2}}} L_{s} t^{\frac{1}{2}}+d\left(\pi+\gamma_{k}\right) t\right]+O\left(e^{-\frac{R_{s}^{2} \cos ^{2}\left(\frac{\gamma_{k}}{2}\right)}{4 t}}\right)+O\left(e^{-\frac{\epsilon_{2}^{2}}{4 t}}\right) .
$$

4. Putting all the estimates together, we arrive at (4.1) with

$$
\delta_{s}=\min \left\{\frac{1}{4} R_{s}^{2} \cos ^{2}\left(\frac{\gamma_{k}}{2}\right), \frac{\epsilon_{0}^{2}}{4}, \frac{\epsilon_{1}^{2}}{4}, \frac{\epsilon_{2}^{2}}{4}\right\} .
$$

In the rest of the proof we show that $\delta_{s}$ satisfies (4.2).

5. By (4.5-4.6), $\epsilon_{0} \geq R_{s} \cos \left(\frac{\gamma_{k}}{2}\right)$ and $\epsilon_{1}=L_{s} \geq R_{s}$. Hence (4.12) reduces to

$$
\delta_{s}=\min \left\{\frac{1}{4} R_{s}^{2} \cos ^{2}\left(\frac{\gamma_{k}}{2}\right), \frac{\epsilon_{2}^{2}}{4}\right\} .
$$

From (4.3), (4.9) and (4.13) we obtain

$$
\delta_{s}=\min \left\{\frac{s^{2}}{16} \sin ^{2}\left(\frac{\pi}{k}\right), \frac{(1-s)^{2}}{16\left[1+\tan \left(\frac{\pi}{k}\right)\right]^{2}}, \frac{s^{2}(1-s)^{2}}{16} \sin ^{2}\left(\frac{2 \pi}{k}\right)\right\}
$$

(recall that $\gamma_{k}=\pi-\frac{2 \pi}{k}$ by (1.13)). One easily checks that for $k=4$ and $0<s \leq s_{k}$

$$
\sin \left(\frac{\pi}{k}\right) \leq \frac{1-s}{1+\tan \left(\frac{\pi}{k}\right)}
$$

and hence, by monotonicity, for $k \geq 4$. One also easily checks that for $k=5$ and $0<s \leq s_{k}$

$$
\sin \left(\frac{\pi}{k}\right) \leq(1-s) \sin \left(\frac{2 \pi}{k}\right)
$$

and hence, by monotonicity, for $k \geq 5$. Therefore we have proved (4.2) for $k \geq 5$. 6 . It remains to prove (4.2) for $k=3,4$. For $k=4$ we have, by (2.3) and (4.13-4.14),

$$
\delta_{s}=\min \left\{\frac{s^{2}}{32}, \frac{(1-s)^{2}}{64}, \frac{s^{2}(1-s)^{2}}{16}\right\} \geq \frac{s^{2}}{16} \min \left\{\frac{1}{2},\left(1-s_{4}\right)^{2}\right\}=\frac{s^{2}}{8}(3-2 \sqrt{2}) .
$$


Finally, for $k=3$ we have, by (2.3) and (4.13),

$$
\begin{aligned}
\delta_{s} & =\frac{s^{2}}{16} \min \left\{\frac{3}{4},\left(\frac{1-s}{s}\right)^{2} \frac{1}{(1+\sqrt{3})^{2}}, \frac{3}{4}(1-s)^{2}\right\} \\
& \geq \frac{s^{2}}{16} \min \left\{\left(\frac{1-s_{3}}{s_{3}}\right)^{2} \frac{1}{(1+\sqrt{3})^{2}}, \frac{3}{4}\left(1-s_{3}\right)^{2}\right\} \\
& =\frac{s^{2}}{16}\left(\frac{1-s_{3}}{s_{3}}\right)^{2} \frac{1}{(1+\sqrt{3})^{2}} \\
& =\frac{s^{2}}{64}(3-\sqrt{5})(2-\sqrt{3}) .
\end{aligned}
$$

For the computation of $\int_{D_{s} \backslash Q_{0}} d x u_{D_{s}}(x ; t)$ it will be convenient to introduce a "model solution' that approximates $u_{D_{s}}$ in one of the branches attached to the basic $k$-gon $Q_{0}$. Consider the half space $H=\left\{\left(x_{1}, x_{2}\right) \in \mathbb{R}^{2}: x_{1}<0\right\}$. Attach one of the $k$ components of $D_{s} \backslash Q_{0}$ to $H$ (recall (1.5)). The resulting set is (see Fig. 6)

$$
H_{s}=\text { interior }\left\{\overline{H \cup\left[\cup_{j \geq 1} \cup_{1 \leq i \leq \frac{1}{k} N(j)} Q_{j, i}\right]}\right\} \text {. }
$$

Let $u_{H_{s}}$ be the solution of (1.1) for $D=H_{s}$. Define

$$
E(t)=\int_{H_{s} \backslash H} d x u_{H_{s}}(x ; t) \quad(t \geq 0) .
$$

Then using Lemma 8 with $D=D_{s}, F=H_{s}, E=H_{s} \backslash H$ and $G=\left\{x \in H_{s}: d(x, E)<\epsilon_{3}\right\}$ with

$$
\epsilon_{3}= \begin{cases}\frac{\sqrt{3}}{4}(1-s) & (k=3) \\ \frac{1}{2}(1-s) & (k \geq 4),\end{cases}
$$

we obtain

$$
\begin{aligned}
\int_{D_{s} \backslash Q_{0}} d x u_{D_{s}}(x ; t) & =k \int_{H_{s} \backslash H} d x u_{D_{s}}(x ; t)+O\left(e^{-\frac{\epsilon_{3}^{2}}{4 t}}\right) \\
& =k E(t)+O\left(e^{-\frac{\epsilon_{3}^{2}}{4 t}}\right) .
\end{aligned}
$$

Thus we must investigate $E(t)$. The advantage of working with $H_{s}$ instead of $D_{s}$ is that the iteration scheme below runs more easily. The following lemma is the key ingredient in the iteration.

Lemma 10 Fix $k \geq 3$. If $0<s \leq s_{k}$, then

$$
\begin{aligned}
& \int_{Q_{1,1}} d x u_{H_{s}}(x ; t)=\frac{2}{\pi^{\frac{1}{2}}}(k-1) s(1-s) t^{\frac{1}{2}} \\
& \quad+\left[-(k-2) c\left(\gamma_{k}\right)-2 c\left(\pi+\gamma_{k}\right)+2(k-2) d\left(\pi+\gamma_{k}\right)\right] t+O\left(e^{-\frac{s^{2} \delta s}{t}}\right),
\end{aligned}
$$

where $\delta_{s}$ satisfies (4.2).

Proof. Partition $Q_{1,1}$ into the following sets (see Fig. 5): 
(i) $k$ kites $U_{\gamma_{k}}\left(s R_{s} \cot \left(\frac{\gamma_{k}}{2}\right)\right)$.

(ii) $2(k-1)$ rectangles $V\left(s R_{s}, s L_{s}\right)$.

(iii) The interior of $Q_{1,1}$ minus (i) and (ii): an open polygon with distance $s \epsilon_{0}$ to $\partial D_{s}$.

(iv) The remainder, which has measure zero.

1. By Lemma 6 , the contribution of (iii) is $O\left(\exp \left(-s^{2} \epsilon_{0}^{2} / 4 t\right)\right)$.

2. The contribution of (i) has two parts: (a) $k-2$ kites contained in wedges $W_{\gamma_{k}}$, (b) 2 kites contained in wedges $W_{\pi+\gamma_{k}}$. For (a) we can use Proposition 1 and Lemma 7 to get a contribution

$$
(k-2)\left[\frac{4}{\pi^{\frac{1}{2}}} s R_{s} \cot \left(\frac{\gamma_{k}}{2}\right) t^{\frac{1}{2}}-c\left(\gamma_{k}\right) t\right]+O\left(e^{-\frac{s^{2} \delta_{s}}{t}}\right) .
$$

The error estimate is the same as in the proof of Lemma 9, but now with space scaled by a factor $s$. For (b), on the other hand, we use Proposition 2 and Lemma 7 to get

$$
2\left[\frac{2}{\pi^{\frac{1}{2}}} s R_{s} \cot \left(\frac{\gamma_{k}}{2}\right) t^{\frac{1}{2}}-\left(c\left(\pi+\gamma_{k}\right)+d\left(\pi+\gamma_{k}\right)\right) t\right]+O\left(e^{-\frac{s^{2} \delta_{s}}{t}}\right) .
$$

3. Finally, the contribution of (ii) follows from Proposition 3 and Lemma 7 and equals

$$
2(k-1)\left[\frac{2}{\pi^{\frac{1}{2}}} s L_{s} t^{\frac{1}{2}}+d\left(\pi+\gamma_{k}\right) t\right]+O\left(e^{-\frac{s^{2} \delta_{s}}{t}}\right) .
$$

Addition of (4.24-4.26) yields the claim (recall (4.3)).

\subsection{Derivation of the functional equation for the heat content}

We are now in a position to derive a functional equation for the heat contained in $H_{s} \backslash H$ in the model solution $u_{H_{s}}$.

Proposition 4 Fix $k \geq 3$. If $0<s \leq s_{k}$, then

$$
\begin{aligned}
E(t)= & (k-1) s^{2} E\left(\frac{t}{s^{2}}\right)+\frac{2}{\pi^{\frac{1}{2}}}(k-1) s(1-s) t^{\frac{1}{2}} \\
& +\left[-(k-2) c\left(\gamma_{k}\right)-2 c\left(\pi+\gamma_{k}\right)+2(k-2) d\left(\pi+\gamma_{k}\right)\right] t+O\left(e^{-\frac{s^{2} \delta_{s}}{t}}\right),
\end{aligned}
$$

where $\delta_{s}$ satisfies (4.2).

Proof. Split

$$
E(t)=\int_{H_{s} \backslash H} d x u_{H_{s}}(x ; t)=\int_{Q_{1,1}} d x u_{H_{s}}(x ; t)+\int_{H_{s} \backslash\left(H \cup Q_{1,1}\right)} d x u_{H_{s}}(x ; t) .
$$

The integral over $Q_{1,1}$ was computed in Lemma 10 and equals the right hand side of (4.27) except for the first term. To estimate the integral over $H_{s} \backslash\left(H \cup Q_{1,1}\right)$, note that this set consists of $k-1$ copies of $H_{s} \backslash H$ scaled by a factor $s$, say $A_{1}, \ldots, A_{k-1}$. Each of these copies has an edge $e_{i}$ connecting it to $Q_{1,1}$. Let $H_{A_{i}}$ be the half space such that $\partial H_{A_{i}} \supset e_{i}$ and 
$H_{A_{i}} \supset Q_{1,1}$. Put $F_{i}=A_{i} \cup H_{A_{i}} \cup e_{i}$. Then $F_{i}$ is a copy of $H_{s}$ scaled by a factor $s$. Therefore we have, by scaling,

$$
\int_{A_{i}} d x u_{F_{i}}(x ; t)=s^{2} \int_{H_{s} \backslash H} d x u_{H_{s}}\left(x ; \frac{t}{s^{2}}\right)=s^{2} E\left(\frac{t}{s^{2}}\right) .
$$

Next, define (see Fig. 6)

$$
G_{i}=\left\{x \in H_{s}: d\left(x, A_{i}\right)<s \epsilon_{3}\right\}
$$

with $\epsilon_{3}$ as in (4.21). This choice guarantees that $G_{1} \backslash A_{1} \subset Q_{1,1}$. We may now apply Lemma 8 with $D=H_{s}, E=A_{i}, F=F_{i}, G=G_{i}$ and $\epsilon=s \epsilon_{3}$. Noting that $\epsilon_{3}^{2} / 4 \geq \delta_{s}$ by (4.14) and (4.21), we obtain

$$
\left|\int_{A_{i}} d x u_{F_{i}}(x ; t)-\int_{A_{i}} d x u_{H_{s}}(x ; t)\right| \leq 2\left|A_{i}\right| e^{-\frac{\left(s \epsilon_{3}\right)^{2}}{4 t}}=O\left(e^{-\frac{s^{2} \delta_{s}}{t}}\right) .
$$

Combine (4.29) and (4.31) to get

$$
\int_{H_{s} \backslash\left(H \cup Q_{1,1}\right)} d x u_{H_{s}}(x ; t)=(k-1) s^{2} E\left(\frac{t}{s^{2}}\right)+O\left(e^{-\frac{s^{2} \delta_{s}}{t}}\right) .
$$

Now use (4.28) and Lemma 10 to get the claim.

\subsection{Proof of the asymptotic expansion for the heat content}

Proposition 4 allows us to derive the expansions formulated in Propositions 5 and 6 below.

Proposition 5 Fix $k \geq 3$ and $s \neq 1 /(k-1)$. Then there exists $\hat{p}_{s}: \mathbb{R} \rightarrow \mathbb{R}$ with period $\log \left(1 / s^{2}\right)$ such that

$$
\begin{aligned}
E(t)= & \hat{p}_{s}(\log t) t^{1-\frac{d_{s}}{2}}+\frac{2}{\pi^{\frac{1}{2}}} \frac{(k-1) s(1-s)}{1-(k-1) s} t^{\frac{1}{2}} \\
& +\left[c\left(\gamma_{k}\right)+\frac{2}{k-2} c\left(\pi+\gamma_{k}\right)-2 d\left(\pi+\gamma_{k}\right)\right] t+O\left(e^{-\frac{s^{2} \delta_{s}}{t}}\right) .
\end{aligned}
$$

Proof. Return to (4.27) in Proposition 4. Abbreviate this relation as

$$
\begin{aligned}
& E(t)=(k-1) s^{2} E\left(\frac{t}{s^{2}}\right)+A_{k} s(1-s) t^{\frac{1}{2}}+B_{k} t+h(t) \\
& A_{k}=\frac{2}{\pi^{\frac{1}{2}}}(k-1) \\
& B_{k}=-(k-2) c\left(\gamma_{k}\right)-2 c\left(\pi+\gamma_{k}\right)+2(k-2) d\left(\pi+\gamma_{k}\right),
\end{aligned}
$$

where $h(t)$ denotes the error term. Next, define $q_{s}(t)$ by

$$
q_{s}(t) t^{1-\frac{d_{s}}{2}}=E(t)-\frac{A_{k} s(1-s)}{1-(k-1) s} t^{\frac{1}{2}}-\frac{B_{k}}{1-(k-1)} t .
$$

Substitute (4.34) into (4.35) to obtain

$$
q_{s}(t) t^{1-\frac{d_{s}}{2}}=(k-1) s^{2} E\left(\frac{t}{s^{2}}\right)-\frac{(k-1) s}{1-(k-1) s} A_{k} s(1-s) t^{\frac{1}{2}}-\frac{k-1}{1-(k-1)} B_{k} t+h(t) .
$$


On the other hand, $t$ replaced by $t / s^{2}$ in (4.35) gives

$$
q_{s}\left(\frac{t}{s^{2}}\right)\left(\frac{t}{s^{2}}\right)^{1-\frac{d_{s}}{2}}=E\left(\frac{t}{s^{2}}\right)+\frac{A_{k} s(1-s)}{1-(k-1) s}\left(\frac{t}{s^{2}}\right)^{\frac{1}{2}}-\frac{B_{k}}{1-(k-1)}\left(\frac{t}{s^{2}}\right) .
$$

But $\left(1 / s^{2}\right)^{1-\frac{d_{s}}{2}}=1 /(k-1) s^{2}$ (recall $(1.9)$ ), and so we conclude from (4.36-4.37) that

$$
q_{s}(t)=q_{s}\left(\frac{t}{s^{2}}\right)+h(t) t^{\frac{d_{s}}{2}-1} .
$$

Next, define $\hat{p}_{s}(\log t)$ by

$$
q_{s}(t)=\hat{p}_{s}(\log t)-\sum_{j=1}^{\infty} h\left(t s^{2 j}\right)\left(t s^{2 j}\right)^{\frac{d s}{2}-1} .
$$

Then (4.38) immediately yields $\hat{p}_{s}(\log t)=\hat{p}_{s}\left(\log \left(t / s^{2}\right)\right)$, so $\hat{p}_{s}$ is $\log \left(1 / s^{2}\right)$-periodic. However, since $h(t)=O\left(\exp \left(-s^{2} \delta / t\right)\right)$, there exists a constant $C$ such that

$$
|h(t)| \leq C e^{-\frac{s^{2} \delta_{s}}{t}} \quad(0<t \leq 1) .
$$

Combining (4.34-4.35) and (4.39-4.40), we get (4.33) with the error term bounded by

$$
C \sum_{j=1}^{\infty}\left(t s^{2 j}\right)^{\frac{d_{s}}{2}-1} e^{-\frac{s^{2}-2 j_{\delta_{s}}}{t}}=O\left(t^{\frac{d_{s}}{2}-1} e^{-\frac{\delta_{s}}{t}}\right) .
$$

Proposition 6 Fix $k=3, \ldots, 8$ and $s=1 /(k-1)$. Then there exists $\hat{p}_{1 /(k-1)}: \mathbb{R} \rightarrow \mathbb{R}$ with period $\log (k-1)^{2}$ such that

$$
\begin{aligned}
E(t)= & \frac{1}{\pi^{\frac{1}{2}}} \frac{k-2}{(k-1) \log (k-1)} t^{\frac{1}{2}} \log \left(\frac{1}{t}\right)+\hat{p}_{\frac{1}{k-1}}(\log t) t^{\frac{1}{2}} \\
& +\left[c\left(\gamma_{k}\right)+\frac{2}{k-2} c\left(\pi+\gamma_{k}\right)-2 d\left(\pi+\gamma_{k}\right)\right] t+O\left(e^{-\frac{\delta_{1 /(k-1)}}{(k-1)^{2} t}}\right) .
\end{aligned}
$$

Proof. The same argument as in the proof of Proposition 5. Because of the log-term one needs to calculate one order further.

Combining Lemma 9, Proposition 5 and (4.22), we obtain the expansions in Theorems 1 and 3. Similarly for Theorem 2 via Proposition 6 . The terms with $c\left(\gamma_{k}\right)$ and $d\left(\pi+\gamma_{k}\right)$ cancel and only those with $c\left(\pi+\gamma_{k}\right)$ remain. Moreover, $p_{s}=k \hat{p}_{s}$ and the error terms are dominated by the largest one carrying $s^{2} \delta_{s} / t$ in the exponent.

\section{$5 \quad$ Study of $p_{s}$}

In this section we prove the properties of $p_{s}$ claimed in Section 1 . 


\subsection{Continuity}

Proposition 7 For all $k \geq 3$ and $0<s \leq s_{k}$ the function $p_{s}$ is continuous.

Proof. Let $x_{0} \in \mathbb{R}$ be arbitrary, and define

$$
\begin{array}{ll}
x_{j} & =x_{0}+2 j \log \left(\frac{1}{s}\right) \\
t_{j} & =e^{x_{j}} \\
d\left(x_{0}\right) & =\limsup _{x \rightarrow x_{0}} p_{s}(x)-\liminf _{x \rightarrow x_{0}} p_{s}(x) .
\end{array}
$$

$1 /(k-1)<s \leq s_{k}$ : By the expansion in Theorem 1, there exists a constant $C$ such that

$$
\left|E_{D_{s}}(t) t^{\frac{d_{s}}{2}-1}-p_{s}(\log t)\right| \leq C t^{\frac{d_{s}-1}{2}} \quad(0<t \leq 1) .
$$

Hence, for all $j \in \mathbb{Z}$ such that $0<t_{j}<1$,

$$
\begin{aligned}
& \limsup _{t \rightarrow t_{j}} p_{s}(\log t) \leq \limsup _{t \rightarrow t_{j}} E_{D_{s}}(t) t^{\frac{d_{s}}{2}-1}+C t_{j}^{\frac{d_{s}-1}{2}} \\
& \liminf _{t \rightarrow t_{j}} p_{s}(\log t) \geq \liminf _{t \rightarrow t_{j}} E_{D_{s}}(t) t^{\frac{d_{s}}{2}-1}-C t_{j}^{\frac{d_{s}-1}{2}} .
\end{aligned}
$$

Since $t \rightarrow E_{D_{s}}(t) t^{\frac{d_{s}}{2}-1}$ is continuous on $(0, \infty)$, it follows from $(5.1)$ and $(5.3)$ that

$$
0 \leq d\left(x_{0}\right) \leq 2 C t_{j}^{\frac{d_{s}-1}{2}}
$$

But $d_{s}>1$, and so we can let $j \rightarrow-\infty$ (i.e., $\left.x_{j} \rightarrow-\infty, t_{j} \downarrow 0\right)$ to conclude that $d\left(x_{0}\right)=0$.

$\underline{s=1 /(k-1)}$ : By the expansion in Theorem 2, there exists a constant $C$ such that

$$
\left|E_{D \frac{1}{k-1}}(t) t^{-\frac{1}{2}}-\frac{1}{\pi^{\frac{1}{2}}} \frac{k(k-2)}{(k-1) \log (k-1)} \log \left(\frac{1}{t}\right)-p_{\frac{1}{k-1}}(\log t)\right| \leq C t^{\frac{1}{2}} \quad(0<t \leq 1) .
$$

Since the first two terms constitute a continuous function, we have by the same argument as in $(5.3-5.4)$ that

$$
0 \leq d\left(x_{0}\right) \leq 2 C t_{j}^{\frac{1}{2}}
$$

Again this yields $d\left(x_{0}\right)=0$ after letting $j \rightarrow-\infty$.

$\underline{0<s<1 /(k-1)}$ : The same argument as in (i) works.

Since $x_{0}$ was arbitrary, we have proved that $d \equiv 0$.

\subsection{Upper bound}

In the following we abbreviate $E_{1}(t)=E_{Q_{0}}(t)$, i.e., the heat content at time $t$ when $D=Q_{0}$ in $(1.1)$. 
Proposition 8 Fix $k=3, \ldots, 8$. If $1 /(k-1)<s \leq s_{k}$, then

$$
p_{s}(\log t) \leq \frac{k}{k-1} \sum_{j \in \mathbb{Z}}\left((k-1) s^{2}\right)^{j+y} E_{1}\left(s^{-2 j-2 y}\right),
$$

where the right hand side is a 1-periodic function in $y=y(s, t)$ defined by

$$
t=s^{-2 y} \text {. }
$$

Moreover,

$$
p_{s} \leq \frac{k}{k-1} \frac{\left|Q_{0}\right|}{1-(k-1) s^{2}}+\frac{4(2 \pi)^{\frac{1}{2}} k^{2} s}{(k-1) s-1}<\infty .
$$

Proof. To prove (5.7-5.8), recall the disjoint composition of $D_{s}$ in (1.5). By fixing the temperature at 1 on all the edges between the constituent $k$-gons, we get an upper bound on the heat content. But in doing so we make all the $k$-gons heat up independently. In a formula:

$$
E_{D_{s}}(t) \leq E_{1}(t)+\sum_{j=1}^{\infty} k(k-1)^{j-1} s^{2 j} E_{1}\left(\frac{t}{s^{2 j}}\right)
$$

where $s^{2 j} E_{1}\left(t / s^{2 j}\right)$ is the heat content at time $t$ of $Q_{0}$ scaled by a factor $s^{j}$. We want to extend the sum to all $j \in \mathbb{Z}$. This goes as follows.

Since

$$
\left|\left\{x \in Q_{0}: d\left(x, \partial Q_{0}\right) \leq \epsilon\right\}\right|=k\left(\epsilon-\epsilon^{2} \tan \left(\frac{\pi}{k}\right)\right) \quad\left(0 \leq \epsilon \leq \frac{1}{2} \cot \left(\frac{\pi}{k}\right)\right),
$$

it follows from [5] Theorem 1.2 that

$$
E_{1}(t) \leq \min \left\{\left|Q_{0}\right|, 4 k(2 \pi t)^{\frac{1}{2}}\right\} \quad(t \geq 0) .
$$

Hence we have

$$
\sum_{j=-\infty}^{0} k(k-1)^{j-1} s^{2 j} E_{1}\left(\frac{t}{s^{2 j}}\right) \leq \frac{4 k^{2}(2 \pi t)^{\frac{1}{2}}}{(k-1)} \sum_{j=0}^{\infty}(k-1)^{-j} s^{-j}=\frac{4 k^{2} s(2 \pi t)^{\frac{1}{2}}}{(k-1) s-1} .
$$

Combining this with $(5.10)$ we find, using that $t^{-\frac{d_{s}}{2}}=(k+1)^{-y}$,

$$
E_{D_{s}}(t) \leq E_{1}(t)+t^{1-\frac{d_{s}}{2}} \frac{k}{k-1} \sum_{j \in \mathbb{Z}}\left((k-1) s^{2}\right)^{j+y} E_{1}\left(s^{-2 y-2 j}\right)+O\left(t^{\frac{1}{2}}\right) .
$$

Since $E_{1}(t)=o\left(t^{1-\frac{d_{s}}{2}}\right)$, the claim in $(5.7)$ now follows with the help of the expansion in Theorem 1.

To prove (5.9), we let $y \in[0,1]$ be arbitrary and partition the sum in (5.7) into two parts:

$$
\begin{aligned}
\frac{k}{k-1} \sum_{j=0}^{\infty}\left((k-1) s^{2}\right)^{j+y} E_{1}\left(s^{-2 j-2 y}\right) & \leq \frac{k}{k-1} \sum_{j=0}^{\infty}\left((k-1) s^{2}\right)^{j+y}\left|Q_{0}\right| \leq \frac{k}{k-1} \frac{\left|Q_{0}\right|}{1-(k-1) s^{2}} \\
\frac{k}{k-1} \sum_{j=1}^{\infty}\left((k-1) s^{2}\right)^{-j+y} E_{1}\left(s^{2 j-2 y}\right) & \leq \frac{4(2 \pi)^{\frac{1}{2} k^{2} s}}{s(k-1)-1}
\end{aligned}
$$

(use that $\left.1 /(k-1)<s \leq s_{k}<1 / \sqrt{k-1}\right)$. The last inequality uses $(5.12)$. 


\subsection{Lower bound}

The derivation of a lower bound for $p_{s}$ requires some further notation. Suppose that $D \subset \mathbb{R}^{2}$ is open and bounded. Suppose that $\partial D_{1}$ is a relatively open subset of $\partial D$ and put $\partial D_{0}=$ $\partial D \backslash \partial D_{1}$. Let $w:(D \cup \partial D) \times[0, \infty) \rightarrow \mathbb{R}$ be the unique (weak) solution of the heat equation

$$
\begin{array}{ll}
\frac{\partial w}{\partial t}=\Delta w & (x \in D, t>0) \\
w(x ; 0)=0 & (x \in D) \\
w(x ; t)=1 & \left(x \in \partial D_{1}, t>0\right) \\
w(x ; t)=0 & \left(x \in \partial D_{0}, t>0\right) .
\end{array}
$$

Then we have the following representation of $w$ :

$$
w(x ; t)=P_{x}\left[T_{D} \leq t, B\left(T_{D}\right) \in \partial D_{1}\right],
$$

with $(B(t): t \geq 0)$ and $T_{D}$ as in Section 3.3. Define the heat content

$$
M_{D}(t)=\int_{D} d x w(x ; t) .
$$

(Note that (3.38-3.39) is a special case of (5.16-5.17): if $\partial D_{0}=\emptyset$, then $u=w$ and $M_{D}=E_{D}$.)

Consider now the basic $k$-gon $Q_{0}$. We choose $\partial D_{0}$ to be the subset of $\partial Q_{0}$ consisting of: (a) 1 (closed) edge of $\partial Q_{0}$, (b) the (closed) middle parts of length $s$ of the remaining $k-1$ edges of $Q_{0}$ (i.e., the parts of $Q_{0}$ where the $k$-gons of the next generation stick on). For $D=Q_{0}$ and this choice of $\partial D_{0}, \partial D_{1}$, abbreviate

$$
M_{1}(t)=M_{Q_{0}}(t) .
$$

Proposition 9 Fix $k=3, \ldots, 8$. If $1 /(k-1)<s \leq s_{k}$, then

$$
p_{s}(\log t) \geq \frac{k}{k-1} \sum_{j \in \mathbb{Z}}\left((k-1) s^{2}\right)^{j+y} M_{1}\left(s^{-2 j-2 y}\right),
$$

where $y=y(s, t)$ is defined as in (5.8). Moreover,

$$
p_{s} \geq \frac{k(1-s)^{2}}{26 \pi^{2}(k-1) s[(k-1) s-1]}>0 .
$$

Proof. To prove (5.20), we keep the temperature at 0 on all the edges of the constituent $k$-gons of $D_{s}$. This way we get a lower bound on the heat content and we again decouple the heat conduction in the different $k$-gons. In a formula:

$$
E_{D_{s}}(t) \geq \sum_{j=1}^{\infty} k(k-1)^{j-1} s^{2 j} M_{1}\left(\frac{t}{s^{2 j}}\right),
$$

where $s^{2 j} M_{1}\left(t / s^{2 j}\right)$ is the heat content at time $t$ of $Q_{0}$ scaled by a factor $s^{j}$ (with the boundary conditions as prescribed above). Next note that $M_{1} \leq E_{1}$. We can therefore use the bound in $(5.12)$ to extend the sum in $(5.22)$ to minus infinity. Namely,

$$
E_{D_{s}}(t) \geq t^{1-\frac{d_{s}}{2}} \frac{k}{k-1} \sum_{j \in \mathbb{Z}}\left((k-1) s^{2}\right)^{j+y} M_{1}\left(s^{-2 y-2 j}\right)+O\left(t^{\frac{1}{2}}\right) .
$$


Comparing this with the expansion in Theorem 1, we conclude that $p_{s}$ satisfies (5.20).

To prove (5.21), we need a lower bound on $M_{1}(t)$. For this we use that

$$
P_{x}\left(T_{D} \leq t, B\left(T_{D}\right) \in \partial D_{1}\right) \geq P_{x}\left(T_{D} \leq t\right)-P_{x}\left(\tau_{\partial D_{0}} \leq t\right),
$$

leading to

$$
w(x ; t) \geq u_{Q_{0}}(x ; t)-P_{x}\left[\tau_{\partial D_{0}} \leq t\right]
$$

and hence

$$
M_{1}(t) \geq E_{1}(t)-\int_{D} d x P_{x}\left[\tau_{\partial D_{0}} \leq t\right] .
$$

Since $Q_{0}$ is convex, we have

$$
u_{Q_{0}}(x ; t) \geq \frac{1}{(\pi t)^{\frac{1}{2}}} \int_{d\left(x, \partial Q_{0}\right)}^{\infty} d q e^{-\frac{q^{2}}{4 t}}
$$

So, the first term in the right hand side of (5.26) can be estimated with the help of (5.11):

$$
\begin{aligned}
E_{1}(t) & \geq \int_{\left[0, \frac{1}{2} \cot \left(\frac{\pi}{k}\right)\right)} d\left|\left\{x \in Q_{0}: d\left(x, \partial Q_{0}\right) \leq \epsilon\right\}\right| \frac{1}{(\pi t)^{\frac{1}{2}}} \int_{\epsilon}^{\infty} d q e^{-\frac{q^{2}}{4 t}} \\
& \geq \int_{[0, \infty)} d \epsilon k\left(\epsilon-\epsilon^{2} \tan \left(\frac{\pi}{k}\right)\right) \frac{1}{(\pi t)^{\frac{1}{2}}} e^{-\frac{\epsilon^{2}}{4 t}} \\
& =\frac{2}{\pi^{\frac{1}{2}}} k t^{\frac{1}{2}}-2 \tan \left(\frac{\pi}{k}\right) k t .
\end{aligned}
$$

To estimate the second term in the right hand side of (5.26), we denote the $k$ line segments of $\partial D_{0}$ by $\partial D_{0, i}(i=1, \ldots, k)$, where the numbering is such that $\left|\partial D_{0, i}\right|=s(i=1, \ldots, k-1)$ and $\left|\partial D_{0, k}\right|=1$. Let $x^{\prime}$ be the orthogonal projection of $x$ onto the line through $\partial D_{0, i}$. Then we have

$$
P_{x}\left[\tau_{\partial D_{0, i}} \leq t\right] \leq \begin{cases}2 e^{-\frac{\left|x-x^{\prime}\right|^{2}}{4 t}} & \left(x^{\prime} \notin \partial D_{0, i}\right) \\ \frac{1}{(\pi t)^{\frac{1}{2}}} \int_{\left|x-x^{\prime}\right|}^{\infty} d q e^{-\frac{q^{2}}{4 t}} & \left(x^{\prime} \in \partial D_{0, i}\right) .\end{cases}
$$

Integrating this over $x \in D$, we obtain

$$
\int_{D} d x P_{x}\left[\tau_{\partial D_{0, i}} \leq t\right] \leq \frac{2}{\pi^{\frac{1}{2}}}\left|\partial D_{0, i}\right| t^{\frac{1}{2}}+16 \pi t
$$

Putting (5.26), (5.28) and (5.30) together, we arrive at

$$
\begin{aligned}
M_{1}(t) & \geq \frac{2}{\pi^{\frac{1}{2}}}(k-1)(1-s) t^{\frac{1}{2}}-2\left(8 \pi+\tan \left(\frac{\pi}{k}\right)\right) k t \\
& \geq \frac{2}{\pi^{\frac{1}{2}}}(k-1)(1-s) t^{\frac{1}{2}}-26 \pi(k-1) t
\end{aligned}
$$

(the last inequality uses that $k \geq 3$ and $\tan \left(\frac{\pi}{k}\right) \leq \sqrt{3}<2 \pi / 3$ ). Note here that only $\partial D_{1}$ contributes to the heat content $M_{1}(t)$, and that $\left|\partial D_{1}\right|=(k-1)(1-s)$.

Let $t_{0}=\left[(1-s)^{2} / 26 \pi^{\frac{3}{2}}\right]^{2}$. Then

$$
M_{1}(t) \geq \frac{1}{\pi^{\frac{1}{2}}}(k-1)(1-s) t^{\frac{1}{2}} \quad\left(0<t \leq t_{0}\right) .
$$


It now follows from (5.23) and (5.32) that

$$
p_{s} \geq \frac{k(1-s)}{\pi^{\frac{1}{2}}} \sum_{\left\{j \in \mathbb{Z}: s^{j+1}>t_{0}^{-\frac{1}{2}}\right\}}((k-1) s)^{j} .
$$

Let $J$ be the unique integer such that $s^{J+1} \geq t_{0}^{-\frac{1}{2}}>s^{J+2}$. Evaluating the geometric series in (5.33), we obtain

$$
\begin{aligned}
p_{s} & \geq \frac{k(1-s)}{\pi^{\frac{1}{2}}} \frac{((k-1) s)^{J+1}}{(k-1) s-1} \\
& \geq \frac{k(1-s)}{\pi^{\frac{1}{2}}(k-1) s} \frac{t_{0}^{\frac{d_{s}-1}{2}}}{(k-1) s-1} \\
& \geq \frac{k(1-s)}{\pi^{\frac{1}{2}}(k-1) s} \frac{t_{0}^{\frac{1}{2}}}{(k-1) s-1}
\end{aligned}
$$

(recall (1.9) and note that $0<t_{0}<1$ ). But the right hand side is precisely the bound claimed.

Propositions 7-9 prove the claims about $p_{s}$ made in Theorems $1-3$, since we have already proved that $p_{s}$ is $\log \left(1 / s^{2}\right)$-periodic.

\section{Partition function: Theorems 5-7}

In this section we list some results for the partition function of our region $D_{s}$ that are the direct analogues of Theorems 1-3 for the heat content. Proofs will be omitted.

Let $\Delta_{D}$ be the Dirichlet Laplace operator for an open set $D \subset \mathbb{R}^{m}(m \geq 2)$. The partition function for $D$ is defined by

$$
Z_{D}(t)=\int_{D} d x p_{D}(x, x ; t),
$$

where $p_{D}(x, y ; t)$ is the corresponding heat kernel. The asymptotic behaviour of $Z_{D}(t)$ as $t \downarrow 0$ is well understood if $D$ is bounded and $\partial D$ is smooth. Moreover, if $m=2, D$ is bounded and connected, and $\partial D$ is a finite polygon, then

$$
Z_{D}(t)=\frac{|D|}{4 \pi t}-\frac{|\partial D|}{8(\pi t)^{\frac{1}{2}}}+\sum_{i=1}^{N} \frac{\pi^{2}-\gamma_{i}^{2}}{24 \pi \gamma_{i}}+O\left(e^{-\frac{r}{t}}\right),
$$

where $\gamma_{1}, \ldots, \gamma_{N}$ are the interior angles at the vertices of $\partial D$, and $r$ is some strictly positive constant depending on $D$ (see [12], [10; p. 44] and [1]). This is to be compared with (1.3).

In the three theorems below, $d_{s}$ is the similarity dimension defined in (1.9) and $r_{s}$ is the error exponent given by (1.14). Recall also (1.6) and (1.10).

Theorem 5 Let $k=3, \ldots, 8$ and $1 /(k-1)<s \leq s_{k}$. Then

$$
Z_{D_{s}}(t)=\frac{\left|D_{s}\right|}{4 \pi t}-q_{s}(\log t) \frac{1}{t^{\frac{d_{s}}{2}}}-\frac{k(1-s)}{1-(k-1) s} \frac{1}{8(\pi t)^{\frac{1}{2}}}-\frac{k-1}{3(k-2)^{2}}+O\left(e^{-\frac{r_{s}}{t}}\right),
$$

with $q_{s}$ a $\log \left(1 / s^{2}\right)$-periodic, continuous and strictly positive function. 
Theorem 6 Let $k=3, \ldots, 8$ and $s=1 /(k-1)$. Then

$$
\begin{aligned}
Z_{D \frac{1}{k-1}}(t)= & \frac{\left|D_{\frac{1}{k-1}}\right|}{4 \pi t}-\frac{k(k-2)}{(k-1) \log (k-1)} \frac{1}{16(\pi t)^{\frac{1}{2}}} \log \left(\frac{1}{t}\right)-q_{\frac{1}{k-1}}(\log t) \frac{1}{t^{\frac{1}{2}}}-\frac{k-1}{3(k-2)^{2}} \\
& +O\left(e^{-\frac{r_{1} /(k-1)}{t}}\right) .
\end{aligned}
$$

with $q_{\frac{1}{k-1}} a \log \left((k-1)^{2}\right)$-periodic and continuous function.

Theorem 7 Let $k=3, \ldots, 8$ and $0<s<1 /(k-1)$ or $k=9,10, \ldots$ and $0<s \leq s_{k}$. Then

$$
Z_{D_{s}}(t)=\frac{\left|D_{s}\right|}{4 \pi t}-\frac{k(1-s)}{1-(k-1) s} \frac{1}{8(\pi t)^{\frac{1}{2}}}-q_{s}(\log t) \frac{1}{t^{\frac{d_{s}}{2}}}-\frac{k-1}{3(k-2)^{2}}+O\left(e^{-\frac{r_{s}}{t}}\right) .
$$

with $q_{s}$ a $\log \left(1 / s^{2}\right)$-periodic and continuous function.

The interpretation of the various terms is similar as in Theorems 1-3. The proof of Theorems 5-7 follows the line of Sections 3-5. The positivity of $q_{s}$ in Theorem 5 follows directly from the results in $[6]$.

A basic tool in the proof is the following. For $t>0$, let $\left(C^{t}(s): s \in[0, t]\right)$ be the standard Brownian bridge on $\mathbb{R}^{2}$ defined by

$$
C^{t}(s)=B(s)-\frac{s}{t} B(t) \quad(s \in[0, t]) .
$$

Let $P_{x x}^{t}$ denote its probability law given $C(0)=x$. Then the analogue of (3.39) reads

$$
p_{D}(x, x ; t)=\frac{1}{4 \pi t} P_{x x}^{t}\left[\left\{C^{t}(s): s \in[0, t]\right\} \cap \partial D=\emptyset\right] \quad(x \in D) .
$$

For example, Lemma 5 has the following analogue.

Lemma 11 For $0<\gamma<\pi$ and $R>0$,

$$
\begin{aligned}
& \frac{1}{4 \pi t} \int_{W_{\pi}(R)} d x P_{x x}^{t}\left[\left\{C^{t}(s): s \in[0, t]\right\} \cap \partial W_{\pi+\gamma} \neq \emptyset\right] \\
& \quad=\frac{R^{2}}{4 \pi t} \int_{0}^{1} d w\left(1-w^{2}\right)^{\frac{1}{2}} e^{-\frac{(R w)^{2}}{t}}-\left[\frac{\pi^{2}-(\pi+\gamma)^{2}}{24(\pi+\gamma)^{2}}+\frac{1}{8(\pi+\gamma)} \cot \left(\frac{\pi^{2}}{\pi+\gamma}\right)\right]+O\left(e^{-\frac{\left[R \sin \left(\frac{\gamma}{2}\right)\right]^{2}}{4 t}}\right) .
\end{aligned}
$$

The computations again rely on the Kontorovich-Lebedev representation of the Dirichlet heat kernel in polar coordinates (see [1]).

\section{References}

[1] M. van den Berg, S. Srisatkunarajah: Heat equation for a region in $\mathbb{R}^{2}$ with a polygonal boundary. J. London Math. Soc. 37 (1988) 119-127. 
[2] M. van den Berg, S. Srisatkunarajah: Heat flow and Brownian motion for a region in $\mathbb{R}^{2}$ with a polygonal boundary. Probab. Th. Rel. Fields 86 (1990) 41-52.

[3] M. van den Berg, P.B. Gilkey: Heat content asymptotics of a Riemannian manifold with boundary. J. Funct. Anal. 120 (1994) 48-71.

[4] M. van den Berg, J.-F. Le Gall: Mean curvature and the heat equation. Math. Z. 215 (1994) 437-464.

[5] M. van den Berg: Heat content and Brownian motion for some regions with a fractal boundary. Probab. Th. Relat. Fields 100 (1994) 439-456.

[6] J. Brossard, R. Carmona: Can one hear the dimension of a fractal? Commun. Math. Phys. 104 (1986) 103-122.

[7] J. Fleckinger, M. Levitin, D. Vassiliev: Heat equation on the triadic von Koch snowflake: asymptotic and numerical analysis. Proc. London Math. Soc. 71 (1995) 372-396.

[8] A. Erdélyi: Tables of Integral Transforms, Vol. 1, McGraw-Hill, New York, 1954.

[9] I.S. Gradshteyn, I.M. Ryzhik: Table of Integrals, Series, and Products (5-th ed.), Academic Press, Boston, 1994.

[10] H.P. McKean, I.M. Singer: Curvature and eigenvalues of the Laplacian. J. Diff. Geom. 1 (1967) 43-69.

[11] B. Simon: Functional Integration and Quantum Physics. Academic Press, New York, 1979.

[12] M. Kac: Can one hear the shape of a drum? Amer. Math. Monthly 73 (1966) 1-23. 
Fig. 1. Three iterations in the construction of $D_{s}$ for $k=3$ (see (1.5) and (1.13)).

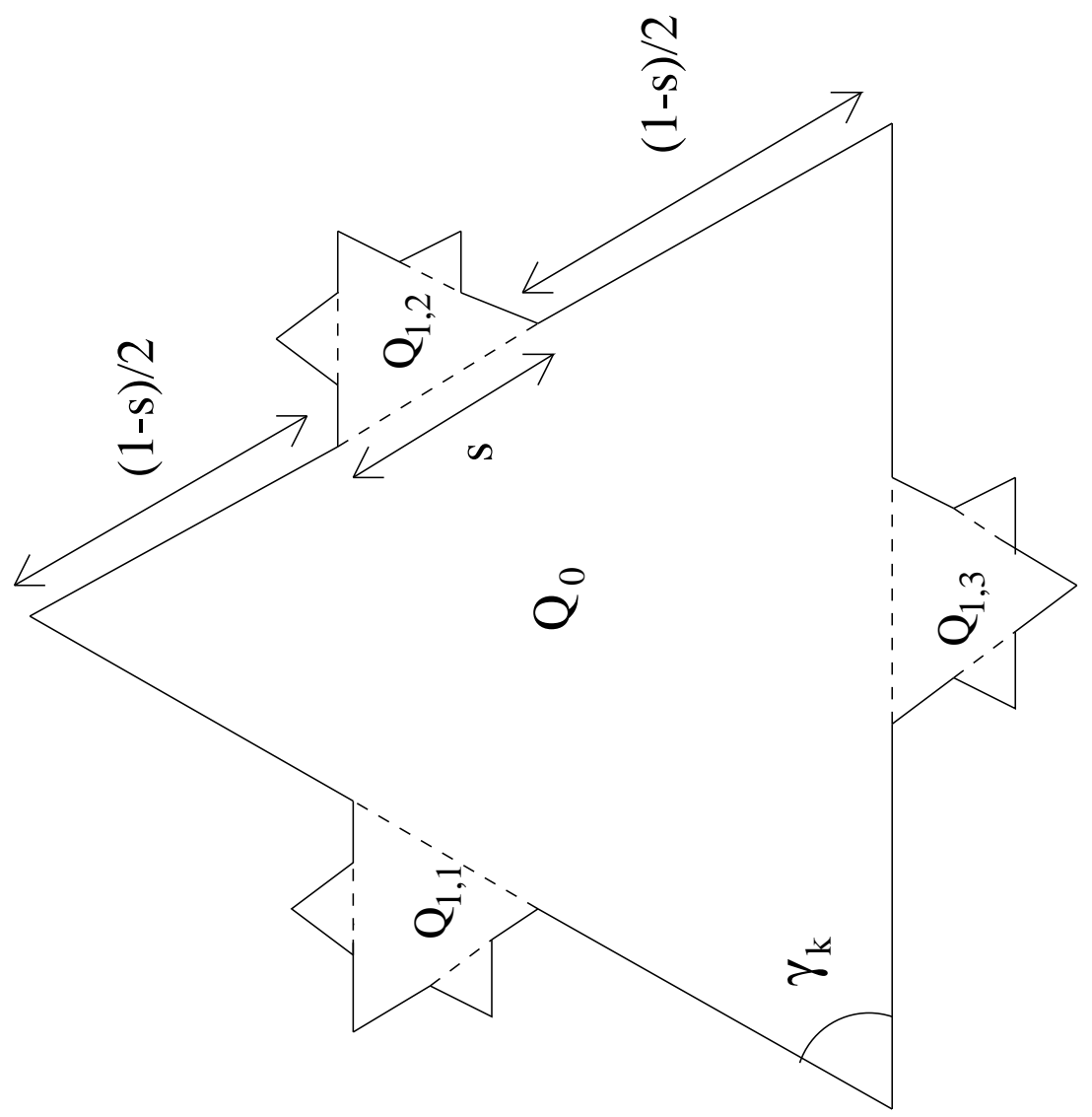


Fig. 2. The kite $U_{\gamma}(R)$ defined in (3.2).

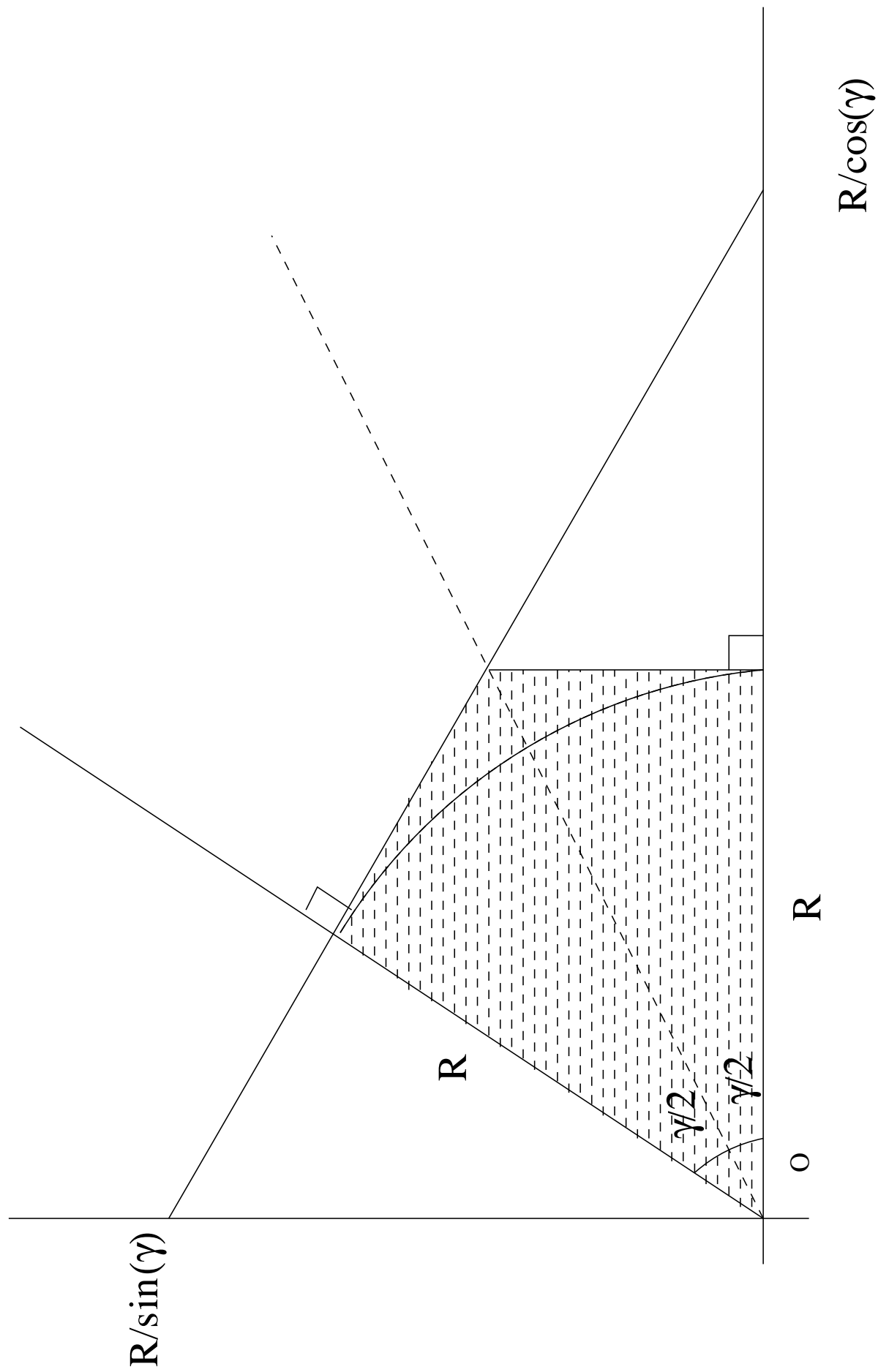


Fig. 3. The sets $C_{\gamma}^{+}(R)$ and $C_{\gamma}^{-}(R)$ defined in (3.53).

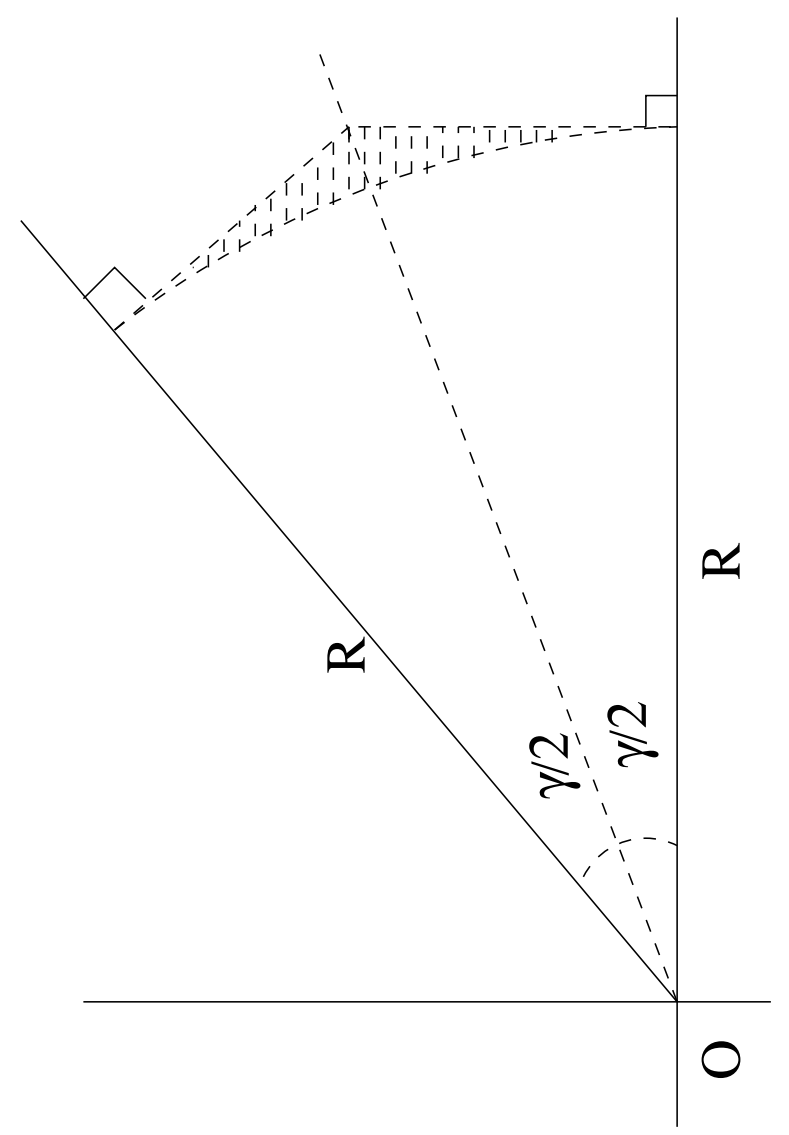


Fig. 4. The sets $D^{+}(R, L)$ and $D^{-}(R, L)$ defined in (3.67).

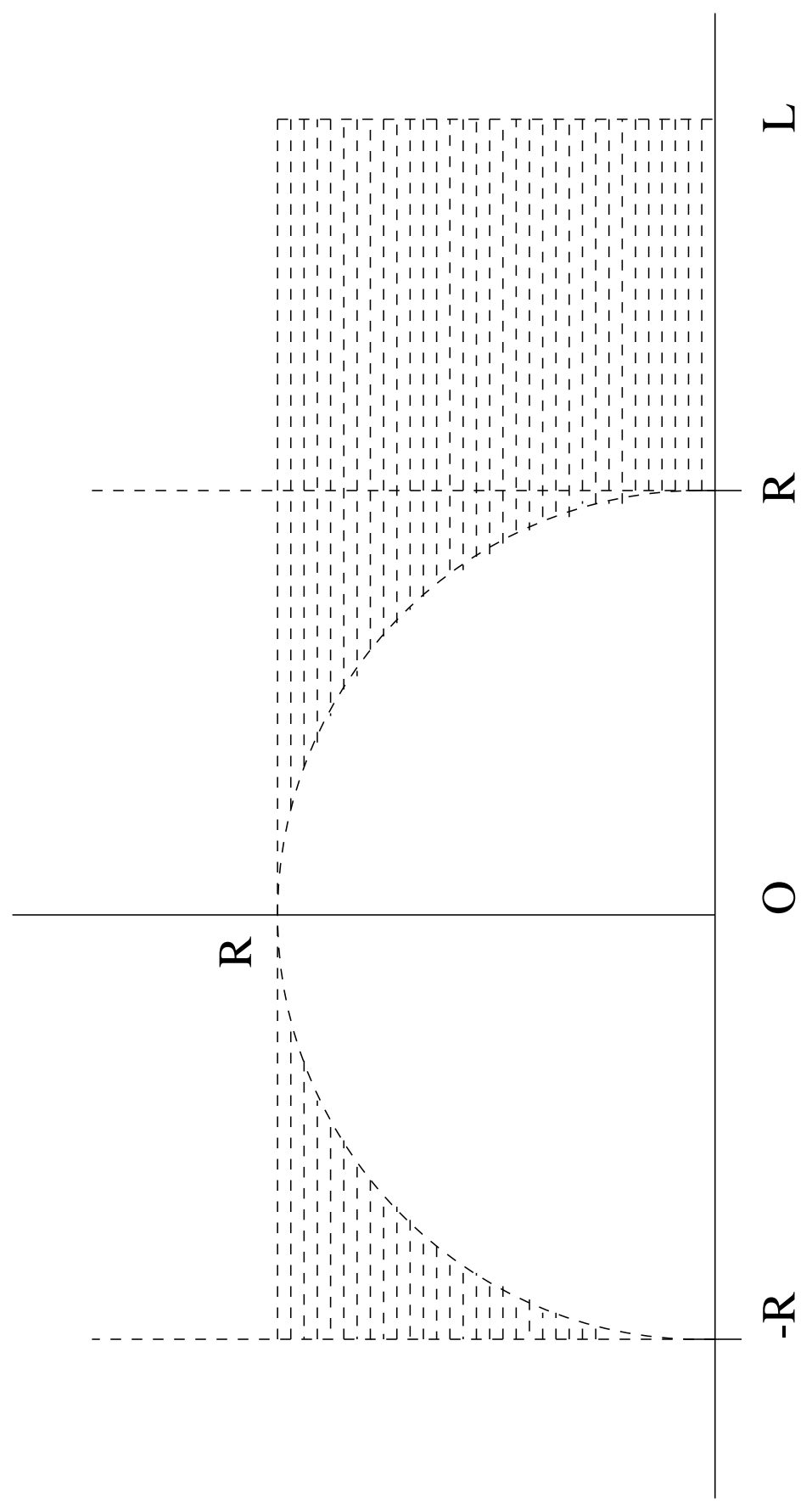


Fig. 5. Partition of $Q_{0}$ for $k=6$ (and for the special case $R_{s}=\frac{s}{2}$ ) in the proof of Lemma 9 in Section 4.1.

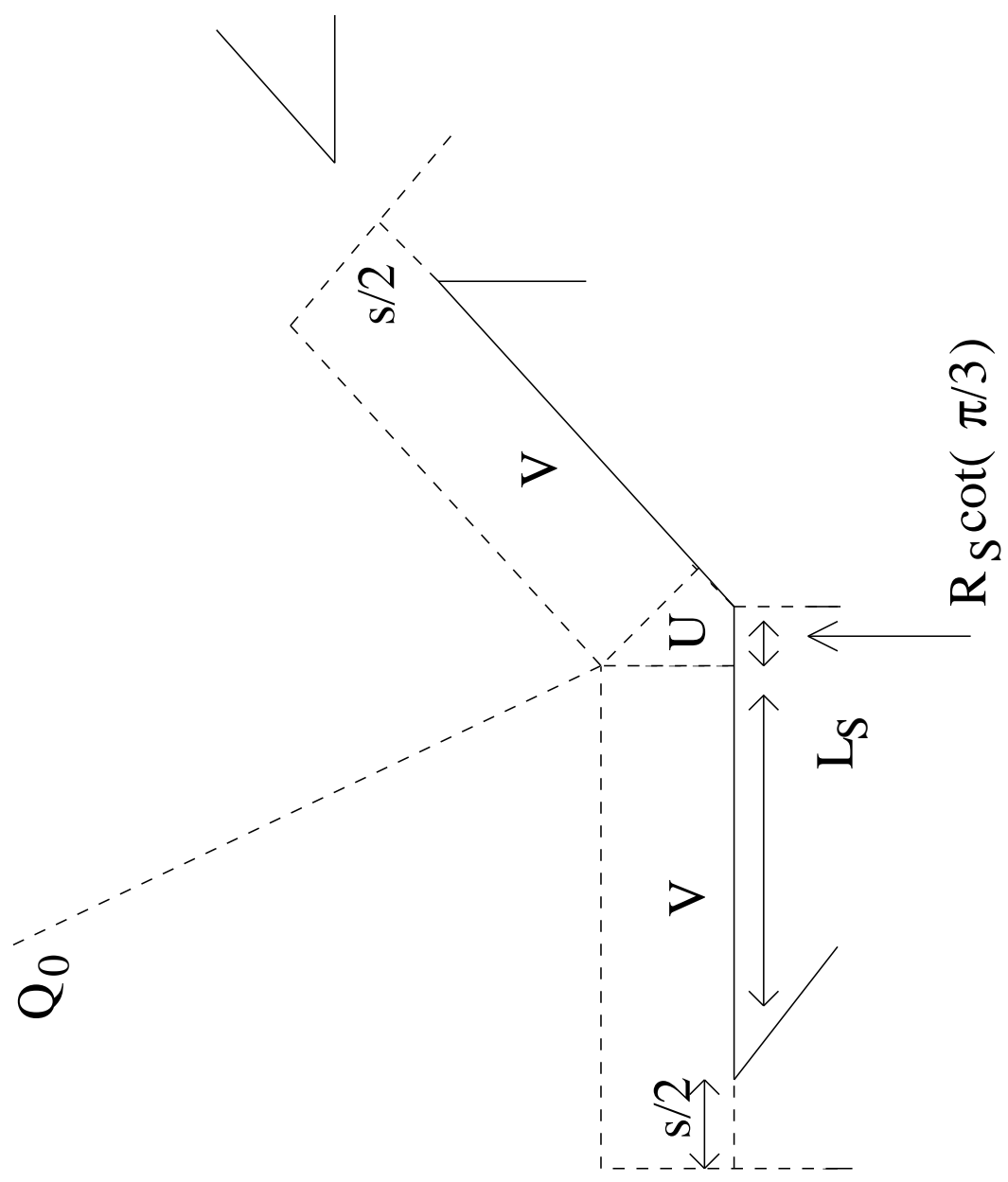


Fig. 6. The set $H_{s}$ for $k=4$ defined in (4.19) (two iterations).

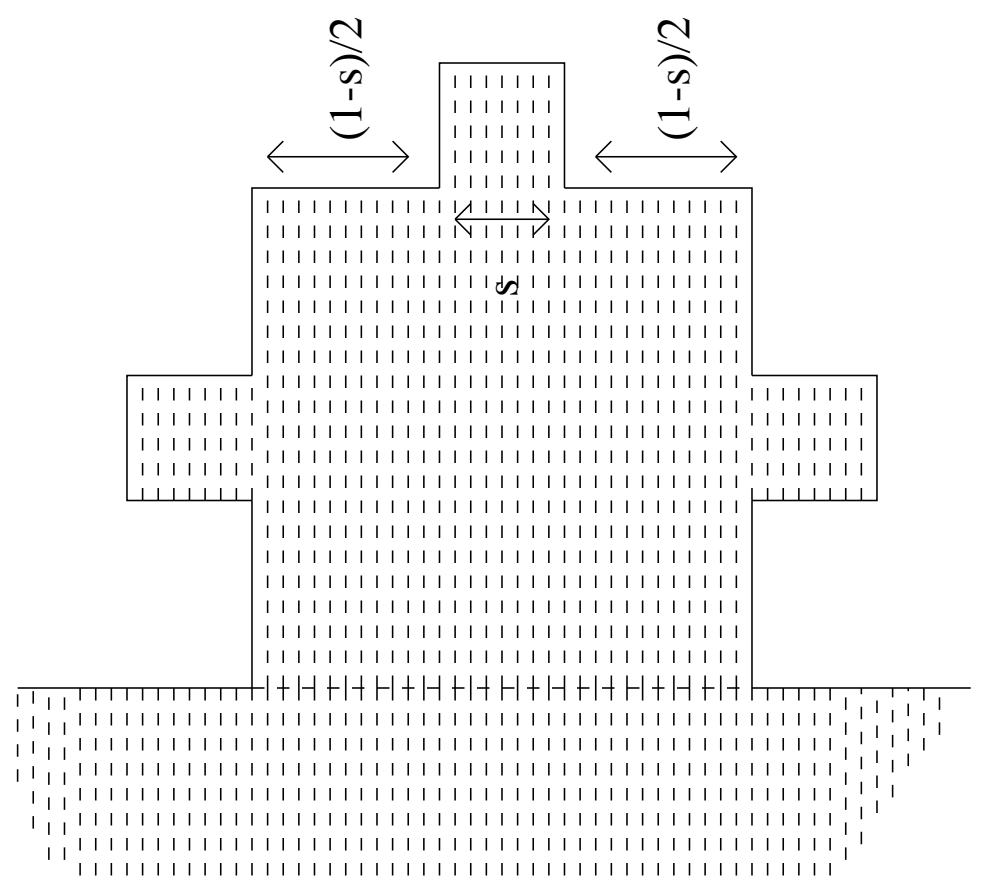

